\title{
Achieving high spatial resolution and high SNR in low-field MRI of hyperpolarised gases with Slow Low Angle SHot
}

\author{
Kajum Safiullin, Cavin Talbot, and Pierre-Jean Nacher ${ }^{\dagger}$ \\ Laboratoire Kastler Brossel, ENS; CNRS; UPMC; 24 rue Lhomond, F-75005 Paris, France.
}

\begin{abstract}
MRI of hyperpolarised gases is usually performed with fast data acquisition to achieve high spatial resolutions despite rapid diffusion-induced signal attenuation. We describe a double-cross k-space sampling scheme suitable for slow low angle shot (SLASH) acquisition and yielding an increased SNR. It consists of a series of anisotropic partial acquisitions with a reduced resolution in the read direction, which alleviates signal attenuation and still provides a high isotropic resolution. The advantages of SLASH imaging over conventional FLASH imaging are evaluated analytically, using numerical lattice calculations, and experimentally in phantom cells filled with hyperpolarised ${ }^{3} \mathrm{He}-$ $\mathrm{N}_{2}$ gas mixtures. Low-field MRI is performed (here $2.7 \mathrm{mT}$ ), a necessary condition to obtain long $T_{2}^{*}$ values in lungs for slow acquisition. Two additional benefits of the SLASH scheme over FLASH imaging have been demonstrated: it is less sensitive to the artefacts due to concomitant gradients and it allows measuring apparent diffusion coefficients for an extended range of times.
\end{abstract}

Keywords: gas MRI; lung MRI; hyperpolarised helium; hyperpolarised gas; apparent diffusion coefficient; ADC.

\section{Introduction}

Two well-identified issues specific to MRI with hyperpolarised (HP) gases strongly affect sequence design and performance. Firstly, the initially available magnetisation is non renewable and thus has to be used up efficiently. Small tip angle excitation, based on the FLASH (Fast Low Angle SHot) sequence [1] is most often used for radial or Cartesian k-space sampling, sometimes with a variable flip angle approach [2]. Secondly, high gas diffusivity leads to rapid signal loss and to image blurring, which sets joint constraints on the acquisition time and on the readout gradient. Increasing the spatial resolution of images implies acquiring more $\mathrm{k}$-space data, using smaller tip angles and faster acquisition rates, at the expense of the signal-to-noise ratio (SNR). Alternative routes for HP gas lung MRI have been evaluated using single-shot sequences, such as EPI [3,4] or RARE [5, 6, 7], or using schemes similar to steady-state free precession imaging $[8,9]$. All these methods yield an increased SNR but are adversely affected by gas diffusion.

Here, we propose a dedicated k-space sampling scheme that can take better advantage of the available signal lifetimes $T_{2}^{*}$ and is more immune to gas diffusion-induced attenuation since acquisition is performed with a moderate applied read gradient: Slow low-angle shot (SLASH) imaging is designed to combine high resolution and high

${ }^{*}$ Current address: Kazan Federal University, Institute of Physics; Kremlevskaya, 18, 420008, Kazan, Russia.

†nacher@lkb.ens.fr, corresponding author.
SNR for HP gas MRI. Slow acquisition imaging of HP gases in lungs is expected to be most efficient at low or moderate applied fields (e.g., 1-100 mT), since high fields (e.g., $1.5 \mathrm{~T}$ and above) induce short values for $T_{2}^{*}$ (of the order of $10 \mathrm{~ms}$ at $3 \mathrm{~T}$ ) due to susceptibility-induced field gradients at alveolar scales [10, 11, 12].

The sensitivity of gas MRI to diffusion-induced attenuation indeed imposes limitations on acquisition parameters and sequence performance, but may also be exploited, in combination with imaging schemes, to probe geometrical restriction of gas diffusion by local sub-voxel structures. Measuring apparent diffusion coefficients (ADC) is a standard characterisation method in various porous media and ADC of HP gas in lungs has been extensively studied in a wide range of situations. Short-range ADC, conveniently measured using short (a few ms) diffusion-sensitising bipolar gradient pulses, probe displacements of ${ }^{3} \mathrm{He}$ atoms on the order of the alveolar diameter. ADC maps associated with diffusion over large distances and long times, which provides more information on the connectivity of lung air spaces at the $\mathrm{cm}$ scale (that of the acinus), are usually obtained with less sensitive stimulated echoes [13] or spin tagging [6] methods. Short- and long-range ADC differ by as much as one order of magnitude; they appear to have different sensitivities and specificities to different lung characteristics and pathologies $[13,14,15,16]$. Using a double-echo imaging sequence can alternatively provide accurate ADC maps, in which the diffusion-sensitising gradient is the imaging read gradient. With SLASH imaging, the probed diffusion time in such double-echo schemes 
can potentially be varied over a broad range of values, especially for low applied magnetic fields and long $T_{2}^{*}$, and so the variation of ADC maps with sensitising time can be studied with a single, high-SNR method.

The article is organised as follows: in the next section, we first theoretically evaluate the effect of free diffusion on signal attenuation, image resolution, and SNR. Given the different impacts of the phase encode and read gradients on signal attenuation, we subsequently introduce a $\mathrm{k}$-space sampling scheme that is suitable for SLASH imaging. We then present numerical lattice simulations used to study the effects of edge enhancement in bounded samples and of the concomitant gradients that play an important role in low field MRI. The experimental 2.7-mT system and the phantom cells used for these in-vitro experiments, and the image reconstruction method are then described. Experimental results are finally presented and discussed: SLASH images are compared to reference FLASH images in terms of image resolution, inter-echo image attenuation, and SNR. Following a summary and a discussion of extensions of double-cross SLASH, supplementary material for the evaluation of diffusion-induced attenuation and of point-spread functions is provided in appendices.

\section{Theoretical considerations}

Evaluating the impact of atomic diffusion on the evolution of magnetisation with the complex interplay of the local conditions (non-uniform gas number density and effective diffusion in heretogeneous porous media or in lung airways), diffusion barriers (walls, obstacles), applied field gradients and RF sequences in gas MR imaging can be a tremendous task. Moreover, in high magnetic field $B_{0}$, the local effect of susceptibility-induced field inhomogeneities in porous media may interfere with that of the applied gradients and anyhow limits the lifetime $T_{2}^{*}$ of transverse local magnetisations to values scaling as $1 / B_{0}[10,11,12]$.

In this section, we address a much simpler situation corresponding to the reported experimental study: hyperpolarised gas with uniform density is enclosed in a non-relaxing container with a negligible amount of paramagnetic $\mathrm{O}_{2}$ and the applied static magnetic field is assumed to be uniform. Hence both the longitudinal and transverse relaxation times are extremely long (they range from minutes in this experiment to several hours or days in optimised situations $[17,18,19])$. These relaxation processes will thus be neglected. In the absence of sizeable radiation damping, the longitudinal magnetisation density $M_{z}(\mathbf{r})$ decreases from its initial value only due to the repeated RF pulses. After $p$ identical non-selective pulses of tip angle $\alpha$, the remaining magnetisation is $M_{z}^{(p)}(\mathbf{r})=\mu_{n} \rho(\mathbf{r}) P \cos ^{p} \alpha$, where $\mu_{n}$ is the magnetic moment of the considered nucleus, $\rho(\mathbf{r})$ the gas density (uniform within the sample, null outside), and $P$ the nuclear polarisation initially prepared by optical pumping. The Bloch equation ruling the evolution of the transverse mag- netisation $M_{+}$is simply (using a complex representation):

$$
\frac{d M_{+}(\mathbf{r}, t)}{d t}=i \omega(\mathbf{r}, t) M_{+}(\mathbf{r}, t)-D \triangle M_{+}(\mathbf{r}, t),
$$

where $D$ is the gas diffusion coefficient. ${ }^{1}$ As usual, Eq. 1 is written for convenience in the rotating Larmor frame and $\omega(\mathbf{r}, t)$ is the local value of the Larmor angular frequency shift resulting from the time-dependent applied field gradients.

The time variation of the applied gradients during a generic 2D projection low-angle shot (or spoiled gradient echo) sequence is represented in Fig. 1. A double-echo acquisition is performed to assess the effect of diffusioninduced magnetisation losses on signal attenuation and on the reconstructed images. The transverse magnetisation

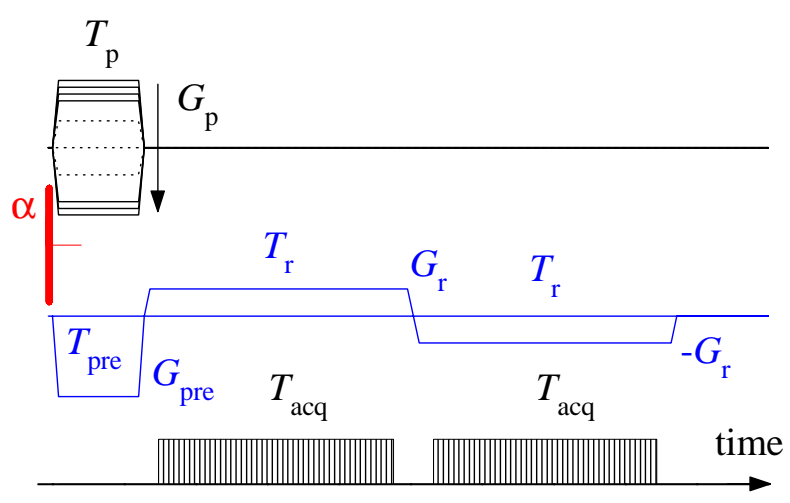

Figure 1: Generic 2D low-angle shot sequence. A nonselective rf pulse is applied (tip angle $\alpha$ ), followed by a phase-encode gradient pulse (area $G_{\mathrm{p}} T_{\mathrm{p}}$ ) and a read preparation gradient pulse (area $G_{\text {pre }} T_{\text {pre }}$ ). A doubleecho acquisition (read gradient areas $\pm G_{\mathrm{r}} T_{\mathrm{r}}$ ) is used to probe diffusion-induced signal losses. Data acquisition is performed during $T_{\text {acq }}$ while gradient intensities are stable. A spoiler gradient pulse (not shown) is applied in the readout direction after the end of the acquisition.

just after tipping is $M_{+}(\mathbf{r}, 0)=i M_{z}^{(p-1)}(\mathbf{r}) \sin \alpha$ if the rf pulse is applied along the $\mathrm{x}$ axis.

With this inital condition, the time evolution of $M_{+}$ during the gradient sequence of Fig. 1 is first evaluated assuming unbounded diffusion (section 2.1) and the resulting values for the image spatial resolution and SNR are derived (section 2.2). Based on these results, a new k-space sampling scheme that allows combining slow acquisition and high resolution is proposed and discussed (section 2.3).

\footnotetext{
${ }^{1}$ For pure ${ }^{3} \mathrm{He}$ gas at 1 atm and temperature $T$, the selfdiffusion coefficient is $D=D_{3}=1.967 \times(T / 300)^{1.71} \mathrm{~cm}^{2} / \mathrm{s}[20,21]$. For a binary mixture of ${ }^{3} \mathrm{He}$ (molar fraction $x$ ) and nitrogen $\mathrm{N}_{2}$, $D^{-1}=x D_{3}^{-1}+(1-x) D_{3-N 2}^{-1}$, where the binary-diffusion coefficient is given by $D_{3-N 2}=0.811 \times(T / 300)^{1.65} \mathrm{~cm}^{2} / \mathrm{s}[20,22]$. This yields $D=1.10 \mathrm{~cm}^{2} / \mathrm{s}$ for the gas mixtures used in this work $(x=0.5$, $T=20^{\circ} \mathrm{C}$ ).
} 


\subsection{Attenuation for free diffusion}

The well-known solution of Eq. 1 for applied uniform gradients of fixed and mutually perpendicular directions $\mathbf{e}_{\mathrm{u}}$ and $\mathbf{e}_{\mathrm{v}}$, and time-varying amplitudes $G_{\mathrm{u}}(t)$ and $G_{\mathrm{v}}(t)$, is given for unbounded diffusion by $\left(\mathbf{e}_{\mathrm{u}}\right.$ and $\mathbf{e}_{\mathrm{v}}$ are unit vectors, $\mathrm{u}$ and $\mathrm{v}$ stand for $\mathrm{x}, \mathrm{y}$ or $\mathrm{z}$ ):

$$
\begin{aligned}
M_{+}(\mathbf{r}, t) & =M_{+}(\mathbf{r}, 0) \exp (-b(t) D) \exp (i \mathbf{k} \cdot \mathbf{r}) \\
\text { with } \quad \mathbf{k}(t) & =\gamma\left(\mathbf{e}_{\mathrm{u}} \int_{0}^{t} G_{\mathrm{u}}\left(t^{\prime}\right) d t^{\prime}+\mathbf{e}_{\mathrm{v}} \int_{0}^{t} G_{\mathrm{v}}\left(t^{\prime}\right) d t^{\prime}\right) \\
\text { and } \quad b(t) & =\int_{0}^{t}\left(k_{\mathrm{u}}^{2}\left(t^{\prime}\right)+k_{\mathrm{v}}^{2}\left(t^{\prime}\right)\right) d t^{\prime}
\end{aligned}
$$

where $\gamma$ is the gyromagnetic ratio $(\gamma=2 \pi \times 32.434 \mathrm{MHz} / \mathrm{T}$ for ${ }^{3} \mathrm{He}$ ). The sums in Eqs. 3 and 4 correspond to the factorisation of the spatial dependencies of the transverse magnetisation, and therefore the effects of the applied gradients of Fig. 1 can be independently evaluated. The wave vector $\mathbf{k}$ associated with the magnetisation helix and the $b$-value leading to the global attenuation factor are obtained by straightforward integration. For simplicity, we assume in the following derivations that rectangular gradient pulses are applied, with negligible ramp times, but more realistic cases have indeed been considered. For the phase-encode gradient, the values of $k$ and $b$ at the end of the gradient pulse are given by:

$$
k_{\mathrm{p}}=\gamma G_{\mathrm{p}} T_{\mathrm{p}} \quad \text { and } \quad b_{\mathrm{p}}=k_{\mathrm{p}}^{2} T_{\mathrm{p}} / 3 .
$$

For the read gradient, $k$ linearly varies between $\pm k_{\mathrm{r}}$, where

$$
k_{\mathrm{r}}=\gamma G_{\text {pre }} T_{\text {pre }}=\gamma G_{\mathrm{r}} T_{\mathrm{r}} / 2,
$$

and the $b$-values $b_{1}$ and $b_{2}$ at the times of the centres of the echoes, $t_{1}=T_{\text {pre }}+T_{\mathrm{r}} / 2$ and $t_{2}=T_{\text {pre }}+3 T_{\mathrm{r}} / 2$, are given by $^{2}$ :

$$
b_{1}=k_{\mathrm{r}}^{2}\left(T_{\mathrm{pre}} / 3+T_{\mathrm{r}} / 6\right) \quad \text { and } \quad b_{2}=b_{1}+k_{\mathrm{r}}^{2} T_{\mathrm{r}} / 3,
$$

while during each echo $i$ ( $i=1$ or 2 ) the $b$-value linearly varies with time as:

$$
b(t)=b_{i}+k_{\mathrm{r}}^{2}\left(t-t_{i}\right) / 3 .
$$

\subsection{Scaling relationships for SNR and resolution}

MR images are reconstructed by Fourier transforms from datasets acquired during the gradient echoes. Due to the boundary condition imposed by the container walls, the simple solutions derived in the previous section cannot be simply linearly combined to infer the exact effect of gas diffusion on the MR images. This will be addressed

\footnotetext{
${ }^{2}$ Here $t_{1}$ and $t_{2}$ are the values of the echo time TE for centred echoes (Eq. 6). This could be adapted in a straightforward way for partial echo acquisition schemes.
}

in Section 3 and in B. Still, evaluating the impact of gas diffusion on image resolution and intensity using a free diffusion assumption is a standard approach $[5,23$, 24]. It provides a convenient way to (over) estimate signal attenuation and to define ranges of acquisition parameters that avoid strong diffusion-induced losses.

The conditions corresponding to $b D \ll 1$ for $b_{\mathrm{p}}, b_{1}$, and $b_{2}$ can be written, in terms of the nominal resolutions $\delta_{\mathrm{p}}=$ $\pi /\left(\gamma G_{\mathrm{p}} T_{\mathrm{p}}\right)$ and $\delta_{\mathrm{r}}=\pi /\left(\gamma G_{\mathrm{r}} T_{\text {acq }}\right)$ in the phase encode and read directions:

$$
\begin{aligned}
T_{\mathrm{p}} & \ll 3 \delta_{\mathrm{p}}^{2} /\left(\pi^{2} D\right), \\
T_{\text {pre }}+T_{\mathrm{r}} / 2 & \ll 3 \delta_{\mathrm{r}}^{2}\left(T_{\mathrm{acq}} / T_{\mathrm{r}}\right)^{2} /\left(\pi^{2} D\right), \\
\text { and } \quad T_{\text {pre }}+3 T_{\mathrm{r}} / 2 & \ll 3 \delta_{\mathrm{r}}^{2}\left(T_{\text {acq }} / T_{\mathrm{r}}\right)^{2} /\left(\pi^{2} D\right) .
\end{aligned}
$$

If these conditions are fulfilled, significant effects of diffusion are avoided. They are conveniently discussed using, for instance, $3 /\left(\pi^{2} D\right)=1.6 \mathrm{~ms} / \mathrm{mm}^{2}$ (the value for pure ${ }^{3}$ He gas). Equation 9 shows that, as derived in [23], millimetre resolutions in the phase-encode direction can be obtained using conventional hardware and $0.5-1 \mathrm{~ms}$ gradient durations. Similar resolutions can be obtained in the read directions as well, provided that ultra-fast (sub-ms) acquisition is performed (Eq. 10), but this is not normally done except in lung MRI of small animals where high resolution is mandatory [25]. For a set read time, the achievable resolution in the image reconstructed from the second echo is indeed coarser, by an amount of order $\sqrt{3}$ (Eq. 11). More details on the evaluation of diffusioninduced attenuation from the chosen imaging parameters and the differences between the effects of the phase-encode and read gradients are provided in $\mathrm{A}$.

A direct consequence of Eqs. 10 and 11 is that the maximum acquisition time scales as $\delta_{\mathrm{r}}^{2}$ (for $T_{\text {pre }} \approx 0$ and $T_{\text {acq }} \approx T_{\mathrm{r}}$ ), hence the sampling rate $f_{\text {samp }}$ and the acquisition bandwidth scale as $\delta_{\mathrm{r}}^{3}$ (for a fixed field of view). As a result, the SNR in the image scales in this limit as:

$$
\mathrm{SNR} \propto \delta_{\mathrm{r}}^{1 / 2} f_{\mathrm{samp}}^{1 / 2} \propto \delta_{\mathrm{r}}^{2} .
$$

The first factor arises from the summation of $\propto 1 / \delta_{\mathrm{r}}$ contributions to the noise in the Fourier transform, the second from the SNR in the time domain [26, chap. 4]. Of course, this relation holds only as long as the acquisition time has the maximum value allowed by the condition set by Eq. 10 or 11, depending on the considered echo. If, for instance, the value of $T_{\text {acq }}$ is fixed, $f_{\text {samp }}$ scales as $\delta_{\mathrm{r}}$ hence the SNR only scales as $\delta_{\mathrm{r}}$.

The SNR also depends on the chosen resolution in the phase-encode direction as:

$$
\mathrm{SNR} \propto \delta_{\mathrm{p}}^{1 / 2} \sin \alpha \approx \delta_{\mathrm{p}}
$$

The first factor has the same origin as in Eq. 12, the second one is due to the fact that, for a set field of view, the tip angle has to decrease as the number $N_{\mathrm{p}}$ of acquired lines in the $k$-space increases, so as to keep $(\cos \alpha)^{N_{\mathrm{p}}} \approx$ $1-N_{\mathrm{p}} \alpha^{2} / 2$ constant. 


\section{$2.3 \quad k$-space sampling strategies - the SLASH sequence}

The preceding discussion highlights the different limiting influences of gas diffusion on image resolution and SNR for phase and frequency encoding protocols in FLASH imaging: the SNR penalty resulting from an increased resolution (a smaller pixel size) is much higher in the read direction (Eqs. 12 and 13). Figure 2 illustrates several approaches that may be used to sample a given region of the k-space. For a set value of the phase encode gradient,

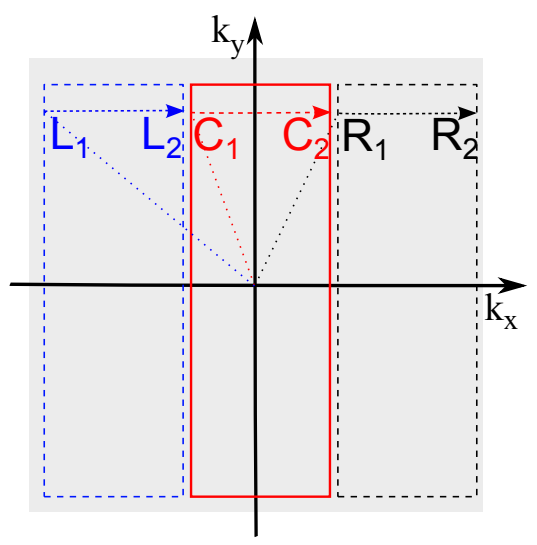

Figure 2: Example of k-space sampling for Cartesian FLASH imaging. The series of values of $G_{\mathrm{p}}$ (along y) and $G_{\mathrm{r}}$ (along x) in the generic sequence of Fig. 1 determine the sampling scheme. An anisotropic resolution is obtained from a rectangular domain centred on the origin. Achieving the full resolution in the read direction requires acquiring data in the off-centred rectangular domains as well, but the diffusion-induced attenuation rate on the path $\mathrm{R}_{1} \rightarrow \mathrm{R}_{2} \rightarrow \mathrm{R}_{1}$ and on the path $\mathrm{L}_{1} \rightarrow \mathrm{R}_{2} \rightarrow \mathrm{L}_{1}$ of the fully scanned $\mathrm{k}$-space are much faster than on the path $\mathrm{C}_{1} \rightarrow \mathrm{C}_{2} \rightarrow \mathrm{C}_{1}$ in the central domain (see text).

the full acquisition for the usual sequence of Fig. 1 corresponds to the paths $\mathrm{L}_{1} \rightarrow \mathrm{R}_{2}$ (first echo) and $\mathrm{R}_{2} \rightarrow \mathrm{L}_{1}$ (second echo), with b-values given by Eqs. 7 and 8 . When only a centred fraction $\kappa$ of the k-space is sampled in the read direction (for instance, $\kappa=1 / 3$ in the example of Fig. 2 along the path $\mathrm{C}_{1} \rightarrow \mathrm{C}_{2}$ and back), a lower resolution is obtained $\left(\delta_{\mathrm{r}}^{\prime}=\delta_{\mathrm{r}} / \kappa\right.$ is larger $)$ and the SNR is increased (here, $1 / \kappa^{2}=9$ times). Recovering the base resolution $\delta_{\mathrm{r}}$ requires sampling through the side regions of the $\mathrm{k}$-space as well.

When a side region is sampled along the same read direction (e.g., $\mathrm{R}_{1} \rightarrow \mathrm{R}_{2} \rightarrow \mathrm{R}_{1}$ for a double echo acquisition), the phase encode direction is still along $\mathrm{y}$, perpendicular to the line $R_{1}-R_{2}$. A straightforward calculation of the $\mathrm{b}$-values at the centres of the sampling paths $\left(b_{1}^{\prime}\right.$ and $b_{2}^{\prime}$ for the first and the second acquisition, respectively), yields:

$$
b_{1}^{\prime}=\kappa^{2} k_{\mathrm{r}}^{2}\left(T_{\mathrm{pre}} / 3+\xi_{1} T_{\mathrm{r}} / 6\right) \text { and } b_{2}^{\prime}=b_{1}^{\prime}+\kappa^{2} k_{\mathrm{r}}^{2} \xi_{2} T_{\mathrm{r}} / 6
$$

with $\xi_{1}=\frac{1+4 \kappa+7 \kappa^{2}}{4 \kappa^{2}}$ and $\xi_{2}=\frac{7+4 \kappa+\kappa^{2}}{2 \kappa^{2}}$.

For $\kappa=1 / 3$ (the example of $k$-space partition depicted in Fig. 2), Eqs. 15 yield $\xi_{1}=7$ and $\xi_{2}=38$. The benefit of sampling along a shorter path for reduced attenuation clearly disappears for an off-centred path such as $\mathrm{R}_{1} \leftrightarrow \mathrm{R}_{2}$. More generally, the benefit of a reduced b-value, that allows performing slow acquisitions with increased SNR, is preserved only when the shortened paths in k-space have no radial component.

This is the case, for instance, of the four partial acquisitions in the sampling scheme of Fig. 3, where the encode direction is successively aligned with the vertical, horizontal, and diagonal directions. In spite of the highly
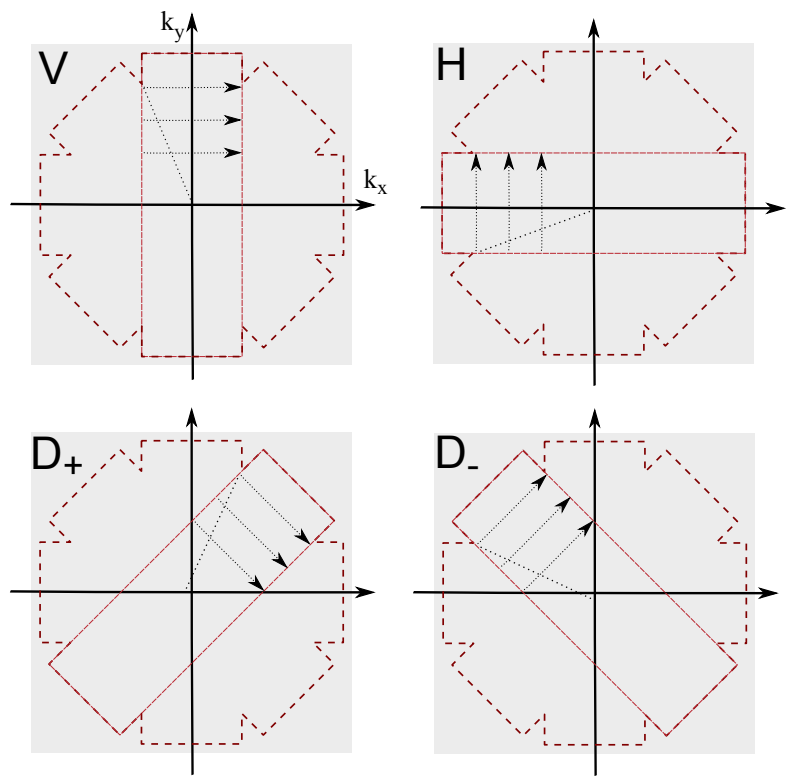

Figure 3: A double-cross acquisition scheme provides a near-isotropic sampling of the k-space (the octagon-like dotted area). A series of four acquisitions using the generic sequence of Fig. 1 with anisotropic resolutions (here, $\kappa=1 / 3$ ) is performed with the phase encode gradient $G_{\mathrm{p}}$ successively along y $(\mathrm{V}), \mathrm{x}(\mathrm{H})$, and the two diagonals $\left(\mathrm{D}_{+}\right.$and $\left.\mathrm{D}_{-}\right)$. The reduced resolution in each read direction allows performing slow acquisition for improved SNR: this is the key feature of the SLASH sequence.

anisotropic resolution associated with each partial acquisition, the k-space is eventually probed almost isotropically (a regular octagon would be obtained for $1 / \kappa=1+\sqrt{2}$ ). Such a Slow Low Angle SHot (SLASH) sequence, that combines partial anisotropic acquisitions according to a suitable pattern (here a double cross overlaying a " + ' and a " $\times$ ' to form an eight-branch star), thus has the potential to provide images with higher resolution and/or SNR than a FLASH sequence when diffusion-induced attenuation is the limiting process.

This double-cross SLASH acquisition scheme used to acquire k-space data might seem similar to that used in 
the PROPELLER sequence (Periodically Rotated Overlapping ParallEL Lines with Enhanced Reconstruction, a.k.a. BLADE, [27]). For both schemes, centred rectangular domains of the k-space are successively acquired for a series of orientations that eventually probe a near-circular domain. As a result, the basic approaches used to perform image reconstruction are the same and therefore some advanced issues regarding, e.g., parallel imaging, partial kspace acquisition, undersampling [28], or image artefacts [29], might be linked. However, the k-space sampling strategies are quite different, with objectives and features that are in sharp contrast. In the PROPELLER scheme, each line of a radial acquisition is replaced by a single-shot EPI-like acquisition of several k-space lines adjacent to a given diameter of the probed domain. Strong frequency encoding and weak phase encoding are thus performed, which would induce strong diffusion-induced attenuation in a highly diffusive sample (e.g., a gas). In contrast, in the double-cross SLASH scheme introduced in this work, each "blade" probed in the k-space is acquired with weak frequency encoding and with independent successive lowangle excitations for the different phase-encoding steps. Each rectangular blade is thus filled with many short segments instead of a few long ones. Reducing the width of the probed k-space domain allows performing slower acquisition for improved SNR without prohibitive signal attenuation arising from diffusion.

\section{$3 \quad$ Numerical lattice calculations}

Solving the Bloch equation (1) for arbitrary conditions falls beyond the scope of this article. However, lattice calculations can be used to illustrate important shortcomings of two standard simplifications that are usually made: assuming free diffusion, and assuming linear field variation (uniform gradients), which jointly yield Eq. 2. The consequences of these simplifications are independently evaluated using different dedicated simple lattice calculations (see B for details and references).

\subsection{Exact 2D simulations}

The presence of container walls (that locally enforce null perpendicular components for the magnetisation currents) may induce strong edge enhancement distortions, that have been observed with hyperpolarised gases in 1D situations $[30,31]$. This has been discussed by several authors in various contexts (see, e.g., [32, 33, 34, 35] and references therein). Exact 2D simulations are introduced here to evaluate edge enhancement effects in our imaging situations, with results given for these simulations in B.

These numerical simulations do not rely on a free diffusion assumption that is indeed not valid when container walls are considered. They are based on an exact calculation of the time evolution of magnetic moments on a $2 \mathrm{D}$ square lattice. Compared to a full 3D simulation, this provides a huge reduction of the computational load and yet captures the essential features of the edge enhancement effects (see B). They include the effects of diffusion and of time-dependent magnetic field gradients. The time evolution is computed by integrating the Bloch equation using a standard Runge-Kutta technique. Actual simulations have been performed for $120 \times 120$ magnetic moments with null normal magnetisation currents at the edges of a square sample. Two situations have been considered: with the applied gradients aligned with the sides or with the diagonals of the sample. They illustrate the influence of the angle of the gradient with the container wall, which spans all possible values in our cylindrical experimental samples.

\subsection{Simplified 3D simulations}

When an imaging or a diffusion sensitising magnetic field gradient is applied, its longitudinal component $\left(G_{\mathrm{u}} \mathbf{e}_{\mathrm{z}}\right.$, the only one so far considered) is inevitably associated with concomitant transverse field components since the total field $\mathbf{B}$ obeys Maxwell's equations $\nabla \cdot \mathbf{B}=0$ and $\nabla \times \mathbf{B}=0$. For simplicity we only consider the transverse gradients used in the axial projection imaging experiments reported here $\left(G_{\mathrm{z}}=0\right)$, hence:

$$
\mathbf{B}(x, y, z)=G_{\mathrm{x}} z \mathbf{e}_{\mathrm{x}}+G_{\mathrm{y}} z \mathbf{e}_{\mathrm{y}}+\left(B_{0}+G_{\mathrm{x}} x+G_{\mathrm{y}} y\right) \mathbf{e}_{\mathrm{z}}
$$

The influence of the concomitant components in low field MRI has been previously described mainly in terms of induced image distortions [36, 37]. Here, two specific issues must be considered, firstly - the $z$ dependence of the local value of $B$ (Eq. 16) reduces the contributions to the NMR signal of elements of the sample lying at large $|z|$ values since no slice selection is performed, and secondly - (as briefly mentioned in [37]) gradient pulses of opposite amplitudes, e.g., $\pm G_{\mathrm{x}}$, do not exactly compensate each other in terms of accumulated phase since spins at a given location precess around slightly different axes with different Larmor frequencies: $\omega_{ \pm}=\gamma\left[\left(B_{0} \pm G_{\mathrm{x}} x\right)^{2}+\left(G_{\mathrm{x}} z\right)^{2}\right]^{1 / 2}$.

The impact of the concomitant gradient components on the time evolution of NMR signals and on the images reconstructed from these signals has been studied using a 3D lattice calculation of the local evolution of magnetisation in an extended sample. The sample shape was the inner volume of the 2-tube cell used in the experiments (see Fig. 4). The lattice parameters were $0.1 \mathrm{~mm}$ in the transverse plane and $1 \mathrm{~mm}$ along the common axis of the cell and the field (100 sites $/ \mathrm{mm}^{3}, \sim 10^{6}$ sites in the sample). For the sake of computational efficiency, the effect of atomic diffusion was coarsely addressed using a free diffusion model (Eqs. 2-4). In contrast, an exact calculation of the local phase of the magnetisation was performed, taking into account the full expression of the magnetic field including concomitant gradient components (Eq. 16). Magnetisation maps and NMR signals were evaluated only 
for the times corresponding to the periodic data acquisition in an imaging sequence (a double-echo FLASH or SLASH acquisition scheme), so that the computational load remained moderate ( $5 \mathrm{~s}$ per phase-encoding step using a desktop computer). Cell length, $B_{0}$ field value, diffusion coefficient, SNR, and imaging parameters were independently varied to study the influence of these factors on the reconstructed images.

\section{Experimental system and meth- ods}

A 1:4 reduced scale test system of a whole-body imaging system prototype [38] was used for low-field MRI at $B_{0}=2.7 \mathrm{mT}\left(\omega_{0} / 2 \pi=87 \mathrm{kHz}\right)$. The static field was produced by five coaxial ring copper wire coils $(29 \mathrm{~cm}$ outer diameter, $26 \mathrm{~cm}$ total length). No cooling was needed below $3 \mathrm{mT}$ and the magnet was driven by a standard laboratory power supply. It was designed to have a uniform field over a 10-cm-diameter sphere, with a computed standard deviation (s.d.) of 15 ppm over this volume. However, imperfect construction of this test system has led to a poorer than expected field homogeneity [38], yielding free induction decay (FID) times $T_{2}^{*} \approx 50 \mathrm{~ms}$ for our 5 -cmlong, 3-cm-diameter samples with first-order shimming of the field by the imaging gradient coils. The transverse gradients were produced by two sets of eight planar coils, inducing linear $\mathrm{x}$ and $\mathrm{y}$ gradients with a computed s.d. of $2.5 \%$ over a 10 -cm-diameter sphere [38]. The z-axis gradient field was produced by four coaxial 15-cm-diameter coils (with a $0.3 \%$ s.d. over $10 \mathrm{~cm}$ ). Standard gradient amplifiers (based on a switched technology) are too noisy at our NMR frequency to be used in the gradient lines. Instead, the gradient coils were driven by linear bipolar power amplifiers (BOP50-8M, Kepco Inc, Flushing NY, USA) interfaced to the sequencer by home-made gradient controllers, for maximum gradient amplitudes of $5.5 \mathrm{mT} / \mathrm{m}\left(G_{\mathrm{x}}, G_{\mathrm{y}}\right)$ and $15 \mathrm{mT} / \mathrm{m}\left(G_{\mathrm{z}}\right)$. Rather long gradient ramp times $(0.2 \mathrm{~ms}$, i.e. more than 100 Larmor precession periods) were chosen to ensure adiabatic response of the magnetisation in spite of the unavoidable changes in the total field orientation due to the concomitant magnetic fields (the Maxwell terms), that may otherwise induce significant magnetisation tipping in large objects at such a low field [37].

NMR was performed using crossed coils. The tipping rf field was generated by a pair of saddle-shaped coils, an exact 1:4 reduced-scale copy of the whole-body $B_{1}$ coils described in ref. [20]. The good rf field homogeneity (checked to be better than $1 \%$ over the samples), combined with long spoiling times (100 ms) between successive encoding steps, ensured a uniform decay of the magnetisation with the repeated rf pulses. The coils were tuned to resonance by a low-loss series capacitor to reduce their impedance and match it that of the in-house rf amplifier (based on a L165 power operational ampli- fier). A pair of rectangular detection coils $\left(7.5 \times 10.5 \mathrm{~cm}^{2}\right.$, $4 \mathrm{~cm}$ apart, with 72 turns each of $25 \times 0.1-\mathrm{mm}$ Litz wire) were tuned as a tank circuit using a C0G capacitor, yielding a quality factor $Q=180$. An active feedback scheme was used to avoid long ring-down dead times following rf pulses and to obtain a broad enough detection bandwidth, suitable for MRI, without SNR penalty [39]. An Apollo Tecmag console was used to manage non-selective rf excitation, preamplifier blanking, and gradient pulses for 2-D imaging. It was also used for data acquisition through a high-impedance, low-noise preamplifier (SR560, Stanford Research Systems, Inc., Sunnyvale CA, USA). All experiments were performed inside a $0.5-\mathrm{mm}$ thick copper Faraday cage providing a 100-fold reduction of picked-up interference noise at our operating frequency [20].

Sets of two and five interconnected parallel glass tubes (50-mm-long) were used as refillable cells (Fig. 4). To reduce nuclear relaxation, the machined surfaces of the end caps have been lined with the same epoxy (Stycast 1266) that was used to glue the glass tubes. Moreover, most of the length of the $1.6 \mathrm{~mm}$ holes drilled through one end cap to let the gas flow between tubes has been lined by $1.6 \times 1.15 \mathrm{~mm}^{2}$ polyethylene catheter. With these precautions, longitudinal relaxation times long enough for imaging experiments with hyperpolarised gas have been obtained $\left(T_{1}: 25\right.$ and 10 minutes for the 2-tube and 5 tube cells).
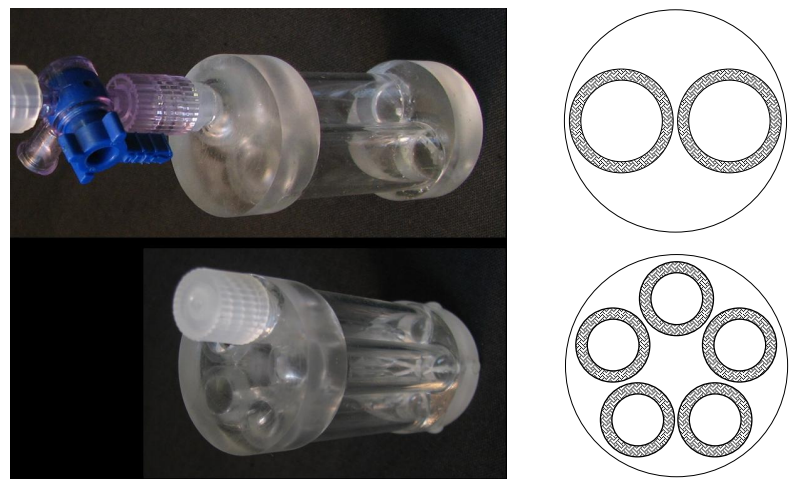

Figure 4: Photographs and schematic cross-sections of the 50-mm-long refillable cells. Upper cell: two 11-mm-i.d. glass tubes, with axes $14.6 \mathrm{~mm}$ apart, are epoxy-glued to plastic (PMMA) end caps. Lower cell: five 7-mm-i.d. tubes, with axes on a 18-mm-diameter circle, are glued to end caps. The tubes are interconnected through the back-side end caps (see text). The two cells have almost the same internal volume $\left(9.5\right.$ and $\left.9.6 \mathrm{~cm}^{3}\right)$.

${ }^{3} \mathrm{He}$ was laser-polarised at low pressure $(\simeq 1 \mathrm{mbar})$ by metastability exchange optical pumping and compressed into a storage cell using an in-house peristaltic compressor [40]. Typical nuclear polarisations of 40-50\% were obtained for gas flow rates of a few standard $\mathrm{cm}^{3}$ per minute. $\mathrm{N}_{2}$ gas was added after compression to obtain a 1:1 mixture $\left(D=1.10 \mathrm{~cm}^{2} / \mathrm{s}\right.$, see footnote 2$)$. The compressor was then used to transfer polarised gas mixture into one 
of the sample cells, with a final pressure usually of order 1 atm. The filled sample cell was then moved in the Earth's field (without additional holding field) from the gas polariser to the MRI setup situated a few meters away, prior to switching the $B_{0}$ field on. The depolarised gas was collected from the sample cells for recycling of the ${ }^{3} \mathrm{He}$ prior to each refill. Sample cells could be refilled several times from a single accumulated batch of polarised gas mixture. Nuclear polarisation was fairly well preserved in the whole process and polarisations up to $40 \%$ were available for imaging.

FID signals were recorded without applied gradients just before and after acquiring an image. The value of the constant tip angle used in each sequence was inferred from the ratio of the FID signal amplitudes. For each image, an additional acquisition was performed without polarised gas for quantitative SNR assessment.

\section{Image reconstruction}

Image reconstruction was performed using compiled inhouse software. The acquired data were trains of double gradient echoes, each echo providing $\mathrm{k}$-space data for one image.

For the FLASH acquisitions, an $N_{\mathrm{p}} \times N_{\mathrm{r}}$ complex matrix was filled using $N_{\mathrm{p}}$ echoes successively acquired for the increasing $k$ values (from $-k_{\mathrm{p}}^{\max }$ to $k_{\mathrm{p}}^{\max }$ ) corresponding to the chosen sequential phase-encoding. The raw data sets were first corrected for the signal attenuation due to tip angle loss: the $n^{\text {th }}$ line was multiplied by $1 /(\cos \alpha)^{n}$. A similar correction scheme was shown recently to improve image quality for radial ${ }^{3} \mathrm{He} \mathrm{MRI}$ as well [41]. An apodisation window function $w_{\mathrm{H}}\left(n, N_{\mathrm{p}}\right) \times w_{\mathrm{H}}\left(p, N_{\mathrm{r}}\right)$ based on the Hanning window $w_{\mathrm{H}}$ was then applied to reduce Gibbs truncation artefacts in the image (see C).

For the double-cross SLASH acquisitions, four $N_{\mathrm{p}} \times$ $N_{\text {r }}$ matrices were first filled with tip-angle corrected data from the four successive partial acquisitions (see Fig. 3). These data sets were then regridded into four $N_{\text {reg }} \times N_{\text {r }}$ matrices in such a way that the new $k$-steps along the phase encode direction were equal to the read $k$-steps $\delta k=$ $\gamma G_{\mathrm{r}} / f_{\text {samp }}$ of the perpendicular band (i.e., along the same physical direction). Since the acquisitions were performed with the same sequences of gradient control voltage values for mutually perpendicular bands, all matrices had the same sizes $\left(N_{\text {reg }} \approx N_{\mathrm{r}} \delta_{\mathrm{r}} / \delta_{\mathrm{p}}\right)$. A slight difference $(10 \%)$ between the two transverse gradient systems resulted in the same difference between the $k$-step values, hence in the FoVs for the two transverse direction. This slight anisotropy was taken into account in the calculations, but for simplicity is not mentioned anymore in the following.

Regridding was performed using a sinc function weighting of the acquired data [42]. This avoided the artefacts that other interpolation methods would unavoidably introduce and involved a negligible computational load for our datasets. The regridded data from the first two par- tial acquisition sets ( $\mathrm{V}$ and $\mathrm{H}$ bands in Fig. 3) was then combined into an $N_{\text {reg }}^{2}$ matrix, in which data of the 4 nonoverlapping regions is the original $\mathrm{V}$ or $\mathrm{H}$ data, whereas data in the central region of the k-space (the intersection of the bands) is the average of $\mathrm{V}$ and $\mathrm{H}$ data weighted by 1 and $(\cos \alpha)^{N_{\mathrm{p}}}$, respectively, to take into account the decreasing SNR resulting from tipping losses. Each of the diagonal bands, $\mathrm{D}_{+}$and $\mathrm{D}_{-}$, was then processed in turn in a similar way, by $45^{\circ}$ rotation, interpolation, and weighted averaging with the combined $\mathrm{V}$ and $\mathrm{H}$ data. Interpolation with a sinc weighting on $2 \mathrm{D}$ grids involved a significant computational load ( $\sim 10$ min with a desktop computer for $N_{\text {reg }}=236$ ). It was used only to check the validity of results obtained using an apodised sinc weighting function for the interpolation. With a Hanning window extending over 60 lattice sites, satisfactory results were obtained with a 100-fold acceleration of the regridding computations.

Following this combination of all acquired data, a radial apodisation window $w\left(\sqrt{n^{2}+p^{2}}, N_{\text {reg }}\right)$ was applied to the octagon-like domain of non-zero data. Different apodisation functions have been tested: the usual Hanning window and broader sine-based apodisation windows. They are defined and compared in $\mathrm{C}$ in which it is demonstrated that Gibbs truncation artefacts are less localised hence less prominent in double-cross SLASH images than in usual FLASH images. Although broader apodisation would be suitable and would provide sharper images for the SLASH images, the same Hanning window was used for all images displayed in the following sections in order to allow a more direct comparison of their resolutions.

The processed k-space data from both FLASH and SLASH acquisitions was then zero-padded to an $N_{\mathrm{FT}}^{2}$ matrix, with $N_{\mathrm{FT}}$ suitably larger than the k-space matrix dimensions $\left(N_{\mathrm{p}}\right.$ and $N_{\mathrm{r}}$, or $N_{\text {reg }}$, respectively) and $2 \mathrm{D}$ discrete Fourier transform (FT) was used to compute a complex image associated with each of the two echoes.

To evaluate the inter-echo signal attenuation and its effect on the shapes and amplitudes of the corresponding images, attenuation maps have been computed for all experiments and for a series of 2D and 3D lattice simulations using the same protocol: a pixel-by-pixel ratio of the image $I_{2}$ reconstructed from the second echo to the image $I_{1}$ from the first echo has been computed for all points with a high enough signal intensity $\left(I_{1}(p, q) \geq I_{1}^{\max } / 10\right.$ was chosen). Whenever necessary, a small translation of $I_{2}$ in the read direction was first performed for experimental data to compensate for slight misregistrations.

\section{Results and discussion}

This section describes and discusses the results of numerical simulations and of imaging experiments using FLASH and double-cross SLASH sequences. After demonstrating how the partial k-space data of the double-cross contributes to the final image resolution, we report on var- 
ious computed contributions to image distortion and attenuation and compare them to the observed inter-echo attenuation maps. Finally, the image SNR is evaluated and analysed by successively considering the noise in the reconstructed images and the previously validated contributions to signal attenuation.

\subsection{Image resolution}

The efficiency of the data combination to yield a highresolution image from the four successive data acquisitions in a SLASH sequence is evidenced by the comparison of the images displayed in Fig. 5 (for each image, the white line is the half-maximum contour). When a single set of
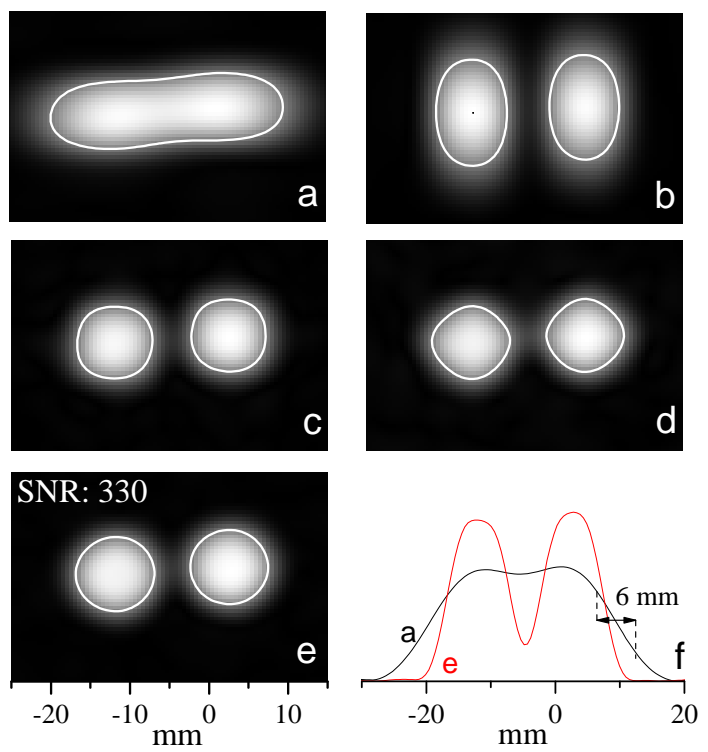

Figure 5: SLASH sequence data from the first echoes are partially or fully used in image reconstruction for the 2tube cell. The half-maximum contour line is superimposed as a white line onto each grayscale image. Top row: a single set of data is used in the image reconstruction (a: $\mathrm{V}$, b: H, see Fig. 3). Middle row: two sets of data with mutually perpendicular encode directions are combined (c: $\left.\mathrm{V}+\mathrm{H}, \mathrm{d}: \mathrm{D}_{+}+\mathrm{D}_{-}\right)$. Bottom row: image e is reconstructed from all data (for SNR evaluation, see Sec. 6.3); Fig. f displays intensity profiles along the common diameter of the tubes for images a (black online) and e (red online). The acquisition parameters are listed in Table 1 $\left(\mathrm{S}_{2}, \mathrm{FoV}_{\mathrm{p}}: 50 \mathrm{~mm}, \mathrm{FoV}_{\mathrm{r}}: 472 \mathrm{~mm}\right)$. Image reconstruction uses $N_{\text {reg }}=236, N_{\mathrm{FT}}=1024$, and the $w_{\mathrm{H}}$ Hanning apodisation function (Eq. 20). Only the central part of the FoV is displayed.

data is used ( $\mathrm{V}$ data in Fig. 5a, $\mathrm{H}$ data in Fig. 5b), an anisotropic resolution of $2 \times 6 \mathrm{~mm}^{2}$ is obtained. Combining two sets of data from perpendicular acquisitions (Figs. $5 \mathrm{c}$ and $5 \mathrm{~d}$ ) improves the quality of the resulting image, but still yields an anisotropic resolution with sharper edges for both perpendicular phase-encoding directions and an overall square- or diamond-like image shape. Combining the whole acquired dataset, that isotropically covers the $\mathrm{k}$-space (after windowing), yields an isotropic resolution in the image (Fig. 5e). The improvement in resolution is also obvious in Fig. 5f, that displays the intensity profiles along the common diameter of the tubes for Fig. 5a, for which $\delta_{\mathrm{r}}=6 \mathrm{~mm}$, and for Fig. $5 \mathrm{e}$.

Series of $2 \mathrm{D}$ projection images using FLASH and SLASH sequences were made, with double-echo acquisition to assess diffusion-induced attenuation. The chosen acquisition parameters are listed in Table 1 . They yield inplane spatial resolutions of $1.8 \times 2.5 \mathrm{~mm}^{2}$ for the FLASH sequence, 1 and $2 \mathrm{~mm}$ for the $\mathrm{S}_{1}$ (high-resolution) and $\mathrm{S}_{2}$ (high-SNR) SLASH sequences.

\begin{tabular}{|l|c|c|c|c|c|c|c|c|c|c|}
\cline { 2 - 11 } \multicolumn{1}{c|}{} & $\alpha$ & $N_{\mathrm{p}}$ & $N_{\mathrm{r}}$ & $d G_{\mathrm{p}}$ & $T_{\mathrm{p}}$ & $G_{\mathrm{r}}$ & $T_{\mathrm{r}}$ & $t_{1}$ & $t_{2}$ & $T_{\mathrm{im}}^{\mathrm{min}}$ \\
\hline $\mathrm{F}$ & $18^{\circ}$ & 24 & 18 & .315 & 2 & 5.5 & 2.5 & 3.6 & 6.3 & 0.12 \\
\hline $\mathrm{S}_{2}$ & $9^{\circ}$ & 100 & 78 & .295 & 2 & 0.62 & 9 & 6.8 & 16 & 1.14 \\
$\mathrm{~S}_{1}$ & $9^{\circ}$ & 124 & 78 & .314 & 3 & 1.23 & 9 & 7.8 & 17 & 1.54 \\
\hline
\end{tabular}

Table 1: Table of acquisition parameters (see Fig. 1) for the compared sequences: FLASH (F, resolution $\left.\delta_{\mathrm{p}}=1.8 \mathrm{~mm}, \quad \delta_{\mathrm{r}}=2.5 \mathrm{~mm}\right)$, high-SNR SLASH $\left(\mathrm{S}_{2}\right.$, $\delta=2 \mathrm{~mm})$, and high-resolution SLASH $\left(\mathrm{S}_{1}, \delta=1 \mathrm{~mm}\right)$. FWHM durations (in $\mathrm{ms}$ ) of the applied gradients (in $\mathrm{mT} / \mathrm{m}$ ) are given. For SLASH acquisitions, actual gradients and resolutions differed by $10 \%$ for the $\mathrm{x}$ and $\mathrm{y}$ directions (see Sec. 5); average values are given. Ramp times lasted $0.2 \mathrm{~ms}$ and acquisitions $\left(f_{\text {samp }}=\mathrm{BW}=10 \mathrm{kHz}\right)$ started after $0.5 \mathrm{~ms}$ of blanking (see footnote 4 ). For the three sequences $T_{\text {pre }}=T_{\mathrm{p}}$ and $G_{\text {pre }}$ were set according to Eq. 6 to obtain centred echoes; $t_{1}$ and $t_{2}$ are the actual echo times for these timings. $T_{\mathrm{im}}^{\mathrm{min}}$ (in seconds) is the minimum imaging time for each sequence, with single echo acquisition and negligible spoiling time.

Examples of the images reconstructed from the first echoes obtained with the FLASH sequence and the highresolution SLASH sequence are displayed in Fig. 6. The resolution of the FLASH images is better in the phase encoding direction (1.8 $\mathrm{mm}$ versus $2.5 \mathrm{~mm}$ in the read direction); this is quite obvious for the narrower tubes of the 5 -tube cell. For both cells, the gain in spatial resolution obtained using the SLASH sequence is quite clear, especially when compared to the resolution in the read direction of the FLASH image (1 mm instead of $2.5 \mathrm{~mm}$ ).

\subsection{Inter-echo attenuation}

The consequences of edge enhancement effects on image attenuation have been studied using the exact 2D lattice simulation in a square cell. For instance, a moderate enhancement of the amplitude in the image $(7 \%)$ is observed near the edges of the sample for the imaging parameters of sequence $\mathrm{S}_{1}$ (see Fig. 14 in B). The attenuation map displays small variations (2.8\% s.d.) of 


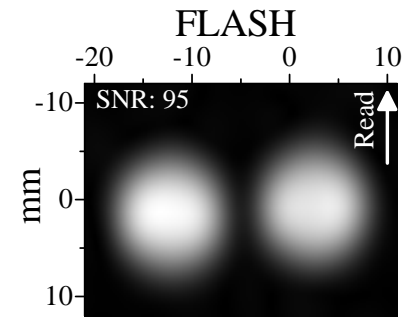

High resolution SLASH
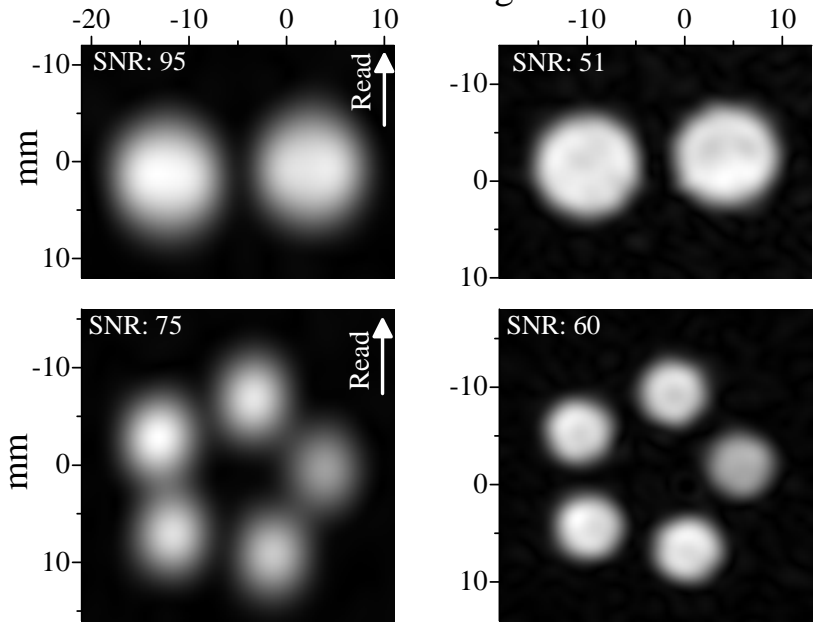

Figure 6: Images of the experimental cells filled with a polarised ${ }^{3} \mathrm{He}-\mathrm{N}_{2}$ mixture, obtained with the FLASH sequence $(\mathrm{F})$ and the high resolution SLASH sequence $\left(\mathrm{S}_{1}\right)$ of Table 1 . The SNR values indicated for each image have been scaled to the available magnetisation (see Sec. 6.3) for quantitative comparison to that of the high SNR SLASH image of Fig. 5 (SNR: 330). Image reconstruction uses $N_{\mathrm{FT}}=256(\mathrm{FLASH})$ or $N_{\mathrm{FT}}=1024$ (SLASH), and the $w_{\mathrm{H}}$ Hanning apodisation function. Only the central parts of the FoV are displayed.

$I_{2} / I_{1}$ over the sample apart from a sharp increase at the very edge; a statistical analysis yields $I_{2} / I_{1}=0.638 \pm 0.018$, whereas the uniform attenuation obtained assuming free diffusion is $I_{2} / I_{1}=0.623$ for the same imaging parameters. For these imaging conditions, the exact diffusion-induced attenuations in the images thus weakly depart from those predicted by a free diffusion model.

The 3D model with free diffusion was therefore used to assess the impact of concomitant gradients on image attenuations. Fig. 7 displays experimental (a) and computed (b) attenuation maps and profiles for the FLASH (F) sequence in which the maximum transverse read gradient was used. With an average attenuation $I_{2} / I_{1}=0.66$,

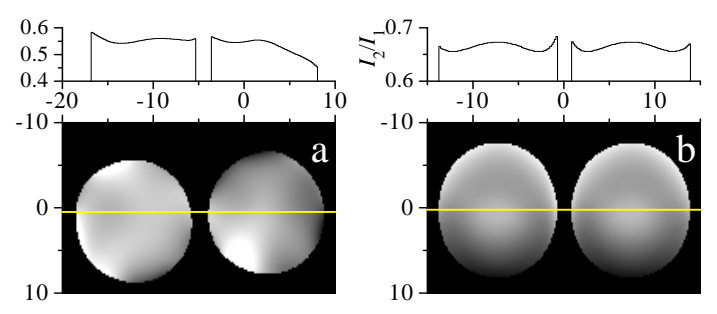

Figure 7: Inter-echo attenuation images and profiles obtained with the FLASH sequence $\mathrm{F}$ in the 2-tube experimental cell (image a) and with the 3D lattice calculation for $D=0$ (image b). The grey-scale images are expanded (0.4-0.6 in image a, 0.6-0.7 in image b) to better display the structures of the attenuation maps. the sole effect of concomitant gradient components accounts for a large fraction of the observed experimental attenuation $I_{2} / I_{1}=0.54$. These average values and the associated standard deviations of $I_{2} / I_{1}$ are listed in Table 2 for the three compared sequences, together with the attenuations induced by free diffusion only and by the combined effect of free diffusion and concomitant gradients.

\begin{tabular}{|l|c|c|c|c|c|c|}
\cline { 2 - 7 } \multicolumn{1}{c|}{} & $R_{\text {cg }}$ & $R_{\mathrm{D}}$ & $R_{3 \mathrm{D}}$ & $R_{T 2 *}$ & $R_{\text {full }}$ & $R_{\exp }$ \\
\hline $\mathrm{F}$ & 0.657 & 0.834 & 0.547 & 0.99 & 0.541 & 0.543 \\
& \pm .019 & & \pm .015 & & \pm .015 & \pm .035 \\
\hline $\mathrm{S}_{2}$ & 0.999 & 0.888 & 0.897 & 0.834 & 0.748 & 0.75 \\
& & & & & & \pm .10 \\
\hline $\mathrm{S}_{1}$ & 0.98 & 0.622 & 0.641 & 0.823 & 0.528 & 0.52 \\
& & & & & & \pm .13 \\
\hline
\end{tabular}

Table 2: Table of computed and measured inter-image attenuation ratios $R=I_{2} / I_{1}$ for the sequences of Table 1 . $R_{\text {cg }}, R_{D}$, and $R_{3 \mathrm{D}}$ are the results of $3 \mathrm{D}$ lattice calculations involving only concomitant gradients, only free diffusion (for $D=1.1 \mathrm{~cm}^{2} / \mathrm{s}$ ), and combining both. $R_{T 2 *}$ evaluates the impact of inhomogeneous broadening (see text), and $R_{\text {full }}$ is the product of $R_{3 \mathrm{D}}$ and $R_{T 2 *} . R_{\exp }$ is the measured attenuation ratio. Standard deviations are given when they exceed $0.5 \%$ of the corresponding ratios.

A last cause for signal attenuation between echoes can become significant if concomitant gradients and diffusioninduced attenuation both have moderate impacts. This is the case for the low-resolution sequence $\mathrm{S}_{2}$, as shown in Fig. 8. The measured attenuation $I_{2} / I_{1}=0.75$ (a) signifi-
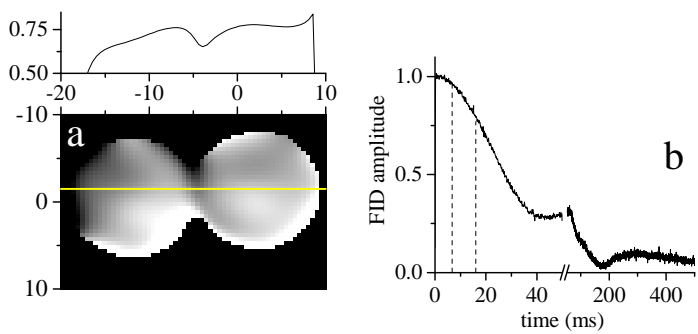

Figure 8: Image a: inter-echo attenuation image (and profile) obtained with the high $\mathrm{SNR}$ sequence $\mathrm{S}_{2}$ in the 2-tube experimental cell. The grey-scale image extends from 0.5 to 0.85. Figure b: amplitude of a FID signal for the same cell (no applied gradient). The times of the echoes of $\mathrm{S}_{2}$ $\left(t_{1}=6.8 \mathrm{~ms}, t_{2}=16 \mathrm{~ms}\right)$ are marked by the vertical dotted lines. The decay time to $1 / e, T_{2}^{*}$, is $\simeq 50 \mathrm{~ms}$.

cantly exceeds that expected from diffusion, $I_{2} / I_{1}=0.89$. Fig. 8b displays a free precession signal in the same cell. The rather short observed signal lifetime is attributed to an imperfect construction of the static field coil system [38]. In the absence of applied imaging gradients, this accounts for significant signal losses at the times $t_{1}$ and $t_{2}$ of the echoes in slow acquisition schemes. The contribution of this inhomogeneous line broadening (from the 
non-linear contributions of the static field imperfections) to the inter-echo attenuation is evaluated using the measured ratio of FID signal amplitudes for each cell shape and set of sequence parameters (given in Table 2) as an independent multiplying factor. With this approach, the expected combined attenuation in the next to the last column of Table 2 satisfactorily accounts for the experimentally observed attenuations listed in the last column of this table.

\subsection{Image SNR}

The SNR of an image is computed as the ratio between the signal amplitude in a fixed domain (region of interest, RoI) within the tube displaying the highest signal and the standard deviation of values over a similar or larger RoI at the same location in a noise image (reconstructed from an identical acquisition without polarised gas). Fig. 9 displays noise data for the same SLASH sequence as in Fig. 5. K-space data (Fig. 9a) is displayed without apodi-
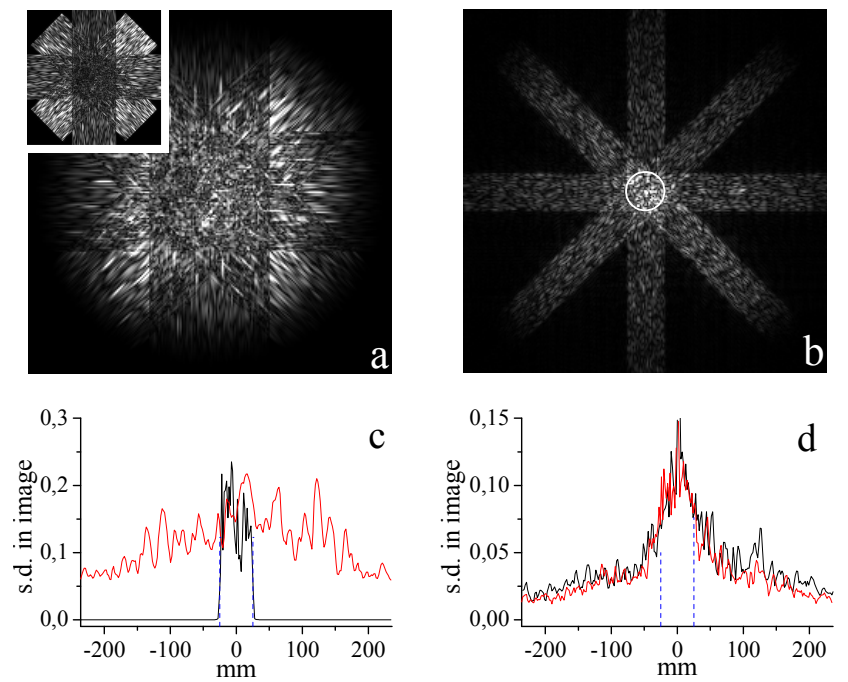

Figure 9: Noise data obtained without polarised gas for the sequence parameters of Fig. 5. Image a: k-space data obtained recombining the 4 bands (inset: data prior to apodisation, see text). Image b: noise image (the FoV is $472 \times 472 \mathrm{~mm}^{2}$, the $50-\mathrm{mm}$ white circle corresponds to the phase-encoding FoV). Figure c: s.d. of data in the image reconstructed from the $\mathrm{V}$ band only (for the broad curve, statistics are taken along the encoding direction). Figure d: s.d. of data along the axes in the fully reconstructed noise image (see text). The dotted lines correspond to the 50-mm phase-encoding FoV.

sation in the inset and with a Hanning apodisation in the main plot. Due to tip angle corrections, successively acquired bands contribute with increasing noise amplitudes (for the last diagonal band, the noise enhancement factor is $\left.1 /\left(\cos 9^{\circ}\right)^{3 \times 25}=2.53\right)$. The reconstructed noise image (Fig. 9b) extends over a field of view much larger than the imaged object and exhibits a highly non-uniform noise pattern outside the actually relevant central region (with the white boundary), which justifies the protocol chosen to compute SNRs.

Fig. 9c displays the noise profiles along the image axes for a noise image reconstructed from the $\mathrm{V}$ band only. In the phase-encode direction, the noise profile is confined in the actually probed 50 -mm-wide FoV thanks to the sinc function weighting used in regridding. In the read direction, the noise profile corresponds to the detection bandwidth of the acquisition system. With the use of active feedback on the detection coil, the bandwidth is broad enough to introduce no distortion in the images $(9 \mathrm{kHz}$ FWHM, corresponding here to $90 \%$ of the 472 -mm-wide FoV). Finally, Fig. 9d displays the noise profiles for the fully reconstructed SLASH image. Isotropy has been recovered, at least in the 50-mm-diameter central region, and it is worthwhile noticing that the noise has been significantly reduced by the averaging process involved in the combination of the multiply-acquired data in the centre of the k-space, in spite of the noise enhancement produced by tip angle corrections. Taking the standard deviation of amplitudes in the noise image of Fig. 9b at the location of one tube of Fig. 5e, a SNR of 330 is obtained for this image.

To account for variations in the gas polarisation between experiments, the SNR value determined for each image has been additionally scaled by the ratio of the available magnetisation and that of the reference image of Fig. 5e, inferred from the FID signals recorded just before each image acquisition. SNR results for six images obtained with the three sequences in the two sample cells are listed in Table 3. With a scaled SNR of the order of 300 , the high-SNR $\left(\mathrm{S}_{2}\right)$ SLASH images have the highest SNR, as expected. Table 3 also lists the computed image

\begin{tabular}{|c|c|c|c||c|c|c|c|}
\cline { 2 - 8 } \multicolumn{1}{c|}{} & SNR & $S_{s c}^{a v}$ & noise & $f_{D}$ & $f_{C G}$ & $A_{\text {pix }}$ & $S_{\text {red }}^{\text {av }}$ \\
\hline $\mathrm{F}, 2 \mathrm{t}$ & 95 & 53 & 0.58 & 0.79 & 0.90 & 4.5 & 54 \\
$\mathrm{~F}, 5 \mathrm{t}$ & 75 & 38 & 0.60 & 0.79 & 0.90 & 4.5 & 39 \\
\hline $\mathrm{S}_{2}, 2 \mathrm{t}$ & 330 & 29 & 0.09 & 0.92 & 1 & 4 & 50 \\
$\mathrm{~S}_{2}, 5 \mathrm{t}$ & 280 & 24 & 0.093 & 0.92 & 1 & 4 & 41 \\
\hline $\mathrm{S}_{1}, 2 \mathrm{t}$ & 51 & 5.5 & 0.11 & 0.67 & 1 & 1 & 52 \\
$\mathrm{~S}_{1}, 5 \mathrm{t}$ & 60 & 5.6 & 0.10 & 0.67 & 1 & 1 & 53 \\
\hline
\end{tabular}

Table 3: Table of scaled SNR and signal values in images for the sequences of Table 1 applied to the 2-tubes (2t) and 5 -tubes (5t) sample cells. Each SNR is evaluated in the tube with the highest signal (see text). Next 2 columns: scaled signals $S_{\mathrm{sc}}^{\mathrm{av}}$, averaged for all tubes, and s.d. of the noise. Next three columns: computed free diffusion attenuation factors $f_{D}$, concomitant gradient loss factors $f_{\mathrm{CG}}$, and nominal pixel areas $A_{\text {pix }}$ (in $\mathrm{mm}^{2}$ ). Last column: reduced amplitudes $S_{\text {red }}^{\text {av }}$ combining the relevant factors (see text).

attenuation factors resulting from signal loss for the first echo due to free diffusion and to concomitant gradients. They are evaluated using the same methods as for inter- 
echo image attenuations in Sect. 6.2 and are thus believed to accurately correspond to the actual attenuation factors.

When comparing the $\mathrm{S}_{2}$ and $\mathrm{S}_{1}$ SLASH sequence results, one should note that the same read timing parameters have been used. This yields much less diffusion attenuation for sequence $\mathrm{S}_{2}$, but the scalings of Eqs. 12 and 13 , that assume that the acquisition parameters are chosen for identical diffusion-induced losses, do not fully apply. The SNR gain of the high-SNR sequence is thus lower that expected: $\times 5-6$ instead of $\times \delta_{\mathrm{r}}^{2} \delta_{\mathrm{p}}=8$. But, given the acquisition parameters of sequence $S_{1}$, the same diffusion induced inter-echo attenuation would be achieved for a readout time $T_{\mathrm{r}}$ of $45 \mathrm{~ms}$ that would imply unacceptable losses from inhomogenous broadening in this experiment (see Fig. 8b).

The benefit of the SLASH sequence with respect to the FLASH acquisition scheme for a set resolution (almost identical for $\mathrm{S}_{2}$ and $\mathrm{F}$ ) mostly arises from the noise reduction associated with a longer acquisition time, here $7.8 \mathrm{~ms}$ instead of $1.8 \mathrm{~ms}\left(\times 2.1^{2}\right)$. The signal reduction resulting from the decrease of the tip angle $(\div 2)$ is actually compensated by the averaging process (of the 4 acquired bands in the centre of the k-space). Altogether, the gain in SNR $(\times 3.6$ from Table 3$)$ is obtained at the expense of an increase of the imaging time (typically $\times 10$ from the values of $T_{\mathrm{im}}^{\mathrm{min}}$ in Table 1). The precise influence of tip angle corrections and of k-space data apodisation on the image noise is found to differ for the SLASH and FLASH reconstructions. The results listed in Table 3 correspond to nearly identical noise characteristics in the time domain.

The last column in Table 3 is the reduced image amplitude $S_{\mathrm{red}}^{\mathrm{av}}=S_{\mathrm{sc}}^{\mathrm{av}} /\left[A_{\mathrm{pix}} f_{D} f_{\mathrm{CG}} \sin \alpha\right]$ (see the caption of the Table). In contrast with the SNR values, that correspond to the highest image intensities among the tubes, the image amplitudes $S_{\mathrm{sc}}^{\mathrm{av}}$ and $S_{\mathrm{red}}^{\mathrm{av}}$ correspond to averages over the 2 or 5 tubes of the cell so that an exact scaling is obtained when using FID signals, that measure the average magnetisations. The 2-tubes cell usually provided balanced intensities (3-7\% difference), whereas a weaker signal (30-45\% below average) was observed in one of the tubes (the right-most one in Fig. 6) for the 5-tubes cell, possibly due to faster relaxation in that tube between cell filling and imaging. Apart from the two low-resolution images in the 5-tubes cell, for which image blurring significantly decreases image intensity, the reduced image amplitude $S_{\text {red }}^{\text {av }}$ is found to be independent of sequence type and parameters, as expected, which confirms that image attenuations are correctly evaluated.

\section{Conclusions and outlook}

We have presented a new k-space sampling scheme consisting of a double-cross pattern of narrow bands with short read segments. It was shown experimentally to produce higher SNR and/or spatial resolution images of ${ }^{3} \mathrm{He}$ gas samples than is achieved with FLASH when a slow ac- quisition scheme (SLASH) is used, as is expected from theoretical considerations on diffusion-induced attenuation. A double-echo acquisition was performed and inter-echo image attenuation was found to be in excellent agreement with the results of numerical computations involving gas diffusion, concommitant gradients, and imperfections of the magnetic field map.

When MRI is performed in conditions of restricted diffusion, such as in lung airspaces, diffusion-induced attenuation involves $\mathrm{ADC}$ values that are smaller than $D$. The associated constraints on the imaging parameters are thus less stringent, but SLASH acquisition with adequate parameters can still be performed to increase SNR or the spatial resolution. MRI of lung airspaces can also be performed using ${ }^{129} \mathrm{Xe}$ instead of ${ }^{3} \mathrm{He}$ as inhaled HP gas. Since the diffusion coefficient of the heavier Xe atom is less than that of $\mathrm{He}$, diffusion-induced attenuation due to imaging gradients is reduced and higher spatial resolutions may be achieved. ${ }^{3}$ However, the SNR of images is generally lower in HP-Xe MRI due to the smaller magnetic moment of the nuclei; therefore, the possible SNR gain of SLASH imaging could be valuable in HP-Xe MRI as well.

The double-cross SLASH scheme could be extended to $3 \mathrm{D}$ acquisition. In a straightforward manner, the k-space volume can be spanned by several sub-volumes, each of them acquired with 2D phase encoding and low-resolution readout in the perpendicular direction, in a way similar to the coverage of the plane by four bands in the double-cross scheme of Fig. 3. More generally, single-shot acquisitions can be performed to reduce imaging time after a phaseencoding step by following any trajectory in the plane perpendicular to that step (it is not restricted to a readout straight line); diffusion-induced attenuation would then only depend on the kinetics of that $2 \mathrm{D}$ read trajectory.

The significant gains in resolution and/or in SNR obtained using a SLASH sequence with a double-cross sampling of the k-space indeed result in longer imaging times. However this time is not as long as if single point imaging (point-by-point spanning of the k-space without readout gradient) was performed, having a total acquisition time up to $N^{2} T_{2}^{*}$ for a $N \times N$ image. In situations where weak inhomogeneous broadening yields long signal lifetimes $T_{2}^{*}$, such as normally met at low magnetic fields, a flexible choice in the sampling pattern (e.g., with more than four $\mathrm{k}$-space bands for an aspect ratio of each band larger than $3: 1$ ) and in the imaging parameters would allow more free-

\footnotetext{
${ }^{3}$ The ADC of ${ }^{129} \mathrm{Xe}$ in normal lungs is typically lower by a factor of 5 than that of ${ }^{3} \mathrm{He}$ [43] whereas the value of $D$ for ${ }^{129} \mathrm{Xe}$ in a $50 \%$ mixture with $\mathrm{N}_{2}$ is $1 / 16$ of that for ${ }^{3} \mathrm{He}$ [22]. Therefore, replacing for instance ${ }^{3} \mathrm{He}$ by ${ }^{129} \mathrm{Xe}$ in our experiments and using the same timings and 11 times higher gradients (because of the 2.75 times lower gyromagnetic ratio of ${ }^{129} \mathrm{Xe}$ ) would yield the same diffusion-induced attenuations with resolutions divided by 4 in Table 1 . In that case, $B_{0}$ should also be increased $(\times 4)$ so that the effects of concomitant gradients on signal attenuation would remain unchanged. This would illustrate the SNR benefit of SLASH compared to FLASH for a $0.5-\mathrm{mm}$ resolution.
} 
dom in deciding between high SNR, high resolution and not-too-long acquisition times. For instance, the acquisition time of the 2-mm resolution sequence $\mathrm{S}_{2}$, unfortunately constrained by the the limited homogeneity of our test system, could be increased to further improve the SNR and also to perform imaging with significant diffusion weighting between the two echoes for ADC measurements at long time scales. To reduce the imaging time, the (near) isotropic SLASH scheme chosen in this work could be replaced by k-space sampling with a suitable ratio between the $k$-steps along two perpendicular directions for a rectangular FoV matching the object shape. Incomplete filling of the $\mathrm{k}$-space, with fewer phase-encoding steps in one direction, could also be used to reduce imaging time at the expense of the resolution in that direction.

The flexibility of SLASH schemes and the achievable gains are ultimately limited by signal lifetimes: seconds in well-shimmed low-field systems, but only milliseconds in lungs at high fields due to susceptibility gradients. Low-field thus provides more flexibility for gas MRI sequence design (RARE, SLASH, or combined strategies). One drawback of low fields arises from the adverse effect of concomitant gradients, but their impact on echo and inter-echo attenuation is strongly reduced for the SLASH sequences that make use of low readout gradient values for frequency encoding. This is fully demonstrated by the systematic use of appropriate lattice simulations that allow assessing precisely the influence of various factors on image distortions and on inter-echo attenuation in multipleecho schemes - a key point for applications to quantitative ADC measurements, performed for instance to probe lung microstructures in normal and diseased animal models or patients.

\section{Acknowledgements}

This work was supported in part by a EU Marie Curie Research and training network (PHeLINet) and by the FPGG (Pierre-Gilles de Gennes) foundation. The authors gratefully thank Emmanuel Baudin who provided computer codes for the exact lattice simulations.

\section{A Sequence parameters and diffusion-induced attenuation}

Different physical processes may affect NMR signals in Low Angle SHot imaging of gases, thereby introducing effective k-space filtering and image blurring if no correction is applied. In this work, data are corrected for tip angle losses (see Sect. 5), which fully preserves the image resolution at the expense of SNR. Regarding the $T_{2}^{*}$-induced and diffusion-induced signal decays, their effects are traditionally evaluated in terms of associated limitations of the achievable resolution [26, chap. 4]. This evaluation is performed assuming, e.g., exponential $T_{2}^{*}$-induced de- cay. If the time-dependent signal attenuation is known (a prerequisite to actually compute a PSF), data correction could be performed as well, thus avoiding the loss of resolution otherwise associated with this attenuation. For diffusion-induced attenuation, we have not used this correction scheme, that remains to be systematically evaluated when edge enhancement plays a role (see B). Neither have we made a straightforward use of the specific results of Ref. [26], as is usually done in work on MRI with polarised gases. Instead, in this Appendix, we explicitly provide standard formulas for diffusion-induced attenuation in a dimensionless form, as well as diagrams in the space of imaging parameters. With the sole assumption that free diffusion prevails, these formulas and diagrams provide means to select suitable imaging parameters for targeted imaging performance (in terms of resolution, SNR, imaging time,...) and to compute PSFs or data correction functions for both the phase encode and the read direction.

It is convenient to introduce the maximum value $G_{\max }$ of the imaging gradients as a scaling quantity and to use dimensionless scaled parameters for gradients: $g_{\mathrm{i}}=$ $G_{\mathrm{i}} / G_{\max }(\mathrm{i}=\mathrm{p}$ for phase-encode, $\mathrm{i}=\mathrm{r}$ for read) for pulse durations: $t_{\mathrm{i}}=T_{\mathrm{i}} / T_{D}$, and for spatial resolutions: $d_{\mathrm{i}}=$ $\delta_{\mathrm{i}} / \delta_{D}$, where

$$
\begin{aligned}
T_{D} & =\left[3 /\left(D \gamma^{2} G_{\max }^{2}\right)\right]^{1 / 3} \\
\delta_{D} & =\pi /\left(\gamma G_{\max } T_{D}\right)
\end{aligned}
$$

$T_{D}$ is the phase-encode time leading to $b_{\mathrm{p}} D=1$ for $G_{\mathrm{p}}=G_{\max }\left(g_{\mathrm{p}}=1\right.$, Eq. 5$)$, and $\delta_{D}$ is the associated nominal resolution.

Regarding the phase-encode gradient, the $b$-value of Eq. 5 is retained to evaluate signal attenuation resulting from gas diffusion during the phase-encode process. The effects of diffusion at later times are overlooked since the average value of the magnetisation within the sample, and hence the signal, remains constant when the gradient is no longer applied. Equation 5 can be written in terms of scaled parameters as:

$$
b_{\mathrm{p}} D=g_{\mathrm{p}}^{2} t_{\mathrm{p}}^{3} \quad \text { and } \quad g_{\mathrm{p}} t_{\mathrm{p}} d_{\mathrm{p}}=1
$$

Figure 10a displays a diagram used to conveniently discuss the results of the choice of sequence parameters on signal attenuation for the phase-encode gradient. The hatched part of the diagram, above the line $g_{\mathrm{p}}=t_{\mathrm{p}}^{-3 / 2}$, corresponds to $b_{\mathrm{p}} D>1$ : this domain of the parameter space is thus to be avoided. The dash-dotted line $g_{\mathrm{p}}=\sqrt{0.1} t_{\mathrm{p}}^{-3 / 2}$ corresponds for instance to $b_{\mathrm{p}} D=0.1$, hence to moderate signal attenuation and $\mathrm{k}$-space filtering. Each dotted line $g_{\mathrm{p}}=\left(d_{\mathrm{p}} t_{\mathrm{p}}\right)^{-1}$ corresponds to the constant phase-encode gradient area that provides the resolution $d_{\mathrm{p}} \delta_{D}$. Along each line, the diffusion-induced loss decreases for decreasing pulse duration down to the value set by the maximum achievable gradient: for $g_{\mathrm{p}}=1, t_{\mathrm{p}}=1 / d_{\mathrm{p}}$ and $b_{\mathrm{p}} D=1 / d_{\mathrm{p}}^{3}$.

On the contrary, diffusion losses associated with the read gradient induce signal attenuation during the whole 
acquisition process, and so the $b$-values of Eq. 7 are used. The conditions $b_{1} D=1$ and $b_{2} D=1$ correspond in Fig. $10 \mathrm{~b}$ to the lines $t_{\mathrm{r}}=2 g_{\mathrm{r}}^{-2 / 3}\left(1+g_{\mathrm{r}}\right)^{-1 / 3}$ and $t_{\mathrm{r}}=2 g_{\mathrm{r}}^{-2 / 3}\left(3+g_{\mathrm{r}}\right)^{-1 / 3}$, respectively. Each dotted line $g_{\mathrm{r}}=2 d^{-1}\left(t_{\mathrm{r}}-t_{\text {blank }}\right)$ still corresponds to the constant resolution $d_{\mathrm{r}} \delta_{D}$, but is now deflected at short times if there is an instrumental blanking time ${ }^{4}$.
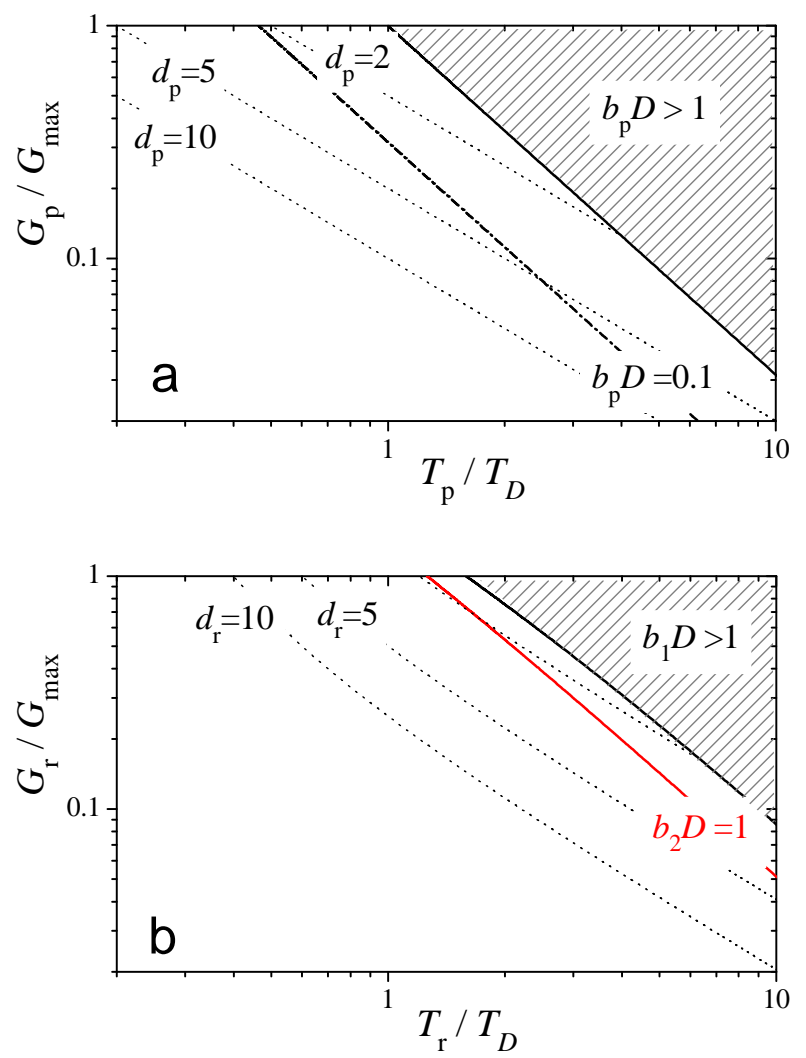

Figure 10: Diagrams used to evaluate the influence of diffusion on signal attenuation for phase-encode (Fig. a) or read (Fig. b) gradients. A point in these diagrams corresponds to a choice of gradient intensity and duration (in units of $G_{\max }$ and $T_{D}$, see text). Signal attenuation decreases for points lying further away from the hatched regions. The dotted lines correspond to fixed resolutions (in units of the scaling resolution $\delta_{D}$ of Eq. 18. In Fig. b, a reduced blanking time $t_{\text {blank }}=0.2$ is used, corresponding to the highest value met in our experiments (for the FLASH sequence).

\section{B Details on the exact 2D model}

Several numerical approaches can be used to solve the full Bloch equations ruling the time evolution of the local magnetisation for situations more complex than that

\footnotetext{
${ }^{4}$ In practice, gradient switching inevitably induces a transient voltage across detection coils, and its slow ringdown at very low NMR frequencies makes it impossible to acquire data immediately after the end of the gradient ramp time.
}

assumed in Sect. 2.1. This is needed for instance when the boundaries of the sample significantly affect the diffusion process, or when magnetisation-dependent magnetic fields introduce additional non-linear contributions to the Bloch equations. These contributions may arise from the radiation damping process that is mediated by the rf coils, or from the direct dipolar couplings between distant parts of a highly magnetised sample.

In the context of hyperpolarised systems, numerical approaches have been used to address these issues in different situations. For instance, a finite-difference treatment of the Bloch equation has been used to simulate ${ }^{3} \mathrm{He}$ diffusion within complex $2 \mathrm{D}$ and $3 \mathrm{D}$ geometries modelling lung airways [44, 45, 46]. Alternatively, several groups have used lattice simulations to study non-linear NMR dynamics in highly magnetised systems [47, 48, 49]. In this work we have used an in-house computer code written in $\mathrm{C}$ that was developed in the framework of such nonlinear NMR studies in liquid ${ }^{3} \mathrm{He}[50,51,52]$. For higher computational efficiency, the effects of magnetic interactions between sites are evaluated in k-space [47] while all other terms in the Bloch equations are computed in the real space.

A realistic 3D representation of our experimental cylindrical samples would involve a very large number of magnetic moments and lattice sites, hence a very heavy computational load (more than $10^{6}$ moments on $10^{7}$ sites, which already requires several days of simulation per phase-encode step on a desktop computer). We thus made two explicit assumptions to tremendously reduce the computational load. Firstly, we assumed that the sample magnetisation did not depend on $z$ by neglecting the concomitant gradients in 2D projection imaging (see Section 3.2). Such an infinite system was exactly represented by a $2 \mathrm{D}$ sample with periodic boundary conditions, i.e. assuming translational invariance along the third $(\mathrm{z})$ dimension. Secondly, we considered a square sample container, with walls situated on lattice sites along the main lattice directions. A rather coarse graining was then sufficient to accurately compute the effects of diffusion throughout the sample.

We have used these $2 \mathrm{D}$ simulations to evaluate the effects of the non-linear terms in the Bloch equation. For parameters corresponding to our experimental imaging conditions, both radiation damping and dipolar couplings were found to play negligible roles in the time evolution of the magnetisation. For our typical magnetisation densities, they induced less than $2 \times 10^{-4}$ of relative changes in the amplitudes of the reconstructed images and have thus been subsequently overlooked. This further simplified the calculations that have been fully performed in the direct space.

When the applied imaging gradients are aligned with the sides of the sample, the factorisation of the spatial dependencies that is obtained for free diffusion (Eqs. 3 and 4 ) is preserved, hence simple 1D solutions are found for $M_{+}$. Examples of results are displayed in Fig. 11 for 
the case where only a phase-encode gradient pulse is used $\left(G_{\mathrm{r}}=0\right)$. The constant value of the signal $S(t)$ following
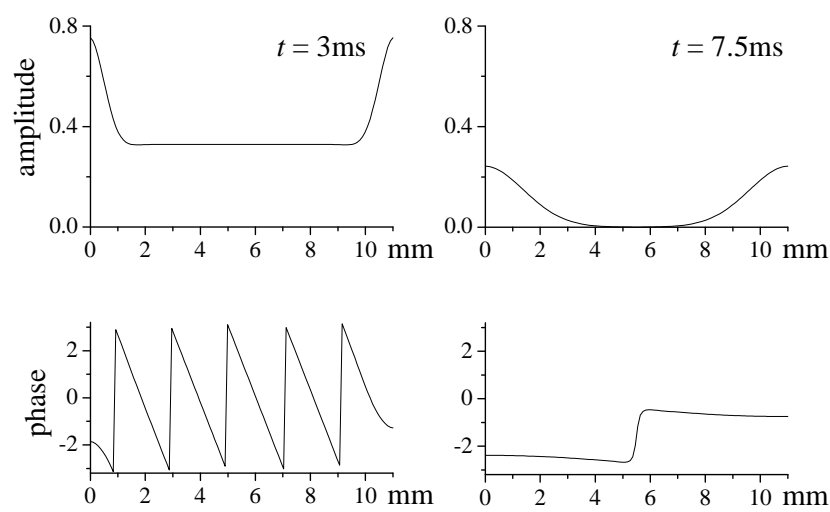

Figure 11: Computed amplitudes (top row) and phases (bottom row) of the local magnetisation $M_{+}(t)$ are plotted as a function of position along the applied gradient $G_{\mathrm{p}}$, assumed here to be parallel to sides of the $11 \times 11 \mathrm{~mm}^{2}$ square sample. The parameters of the computation are set to correspond to those of the SLASH images in Fig. 6: $T_{\mathrm{p}}=3 \mathrm{~ms}, G_{\mathrm{p}}=5 \mathrm{mT} / \mathrm{m}$ (hence $k_{\mathrm{p}}=30 \mathrm{~cm}$ and $\delta_{\mathrm{p}}=1 \mathrm{~mm}$ ), $D=1.1 \mathrm{~cm}^{2} / \mathrm{s}$. Left column (at $t=T_{\mathrm{p}}$ ): magnetisation just at the end of the encoding pulse. Right column (at $\left.t=t_{1}\right)$ : magnetisation at the time of the first read gradient echo in Fig. 6.

the end of the phase encoding, here found to be $0.0531 \times$ $S(0)$ with the chosen phase encoding, results from magnetisation maps that strongly change with time but yield a constant magnetisation average. For the chosen parameters (corresponding to the highest encoding step in the image of Fig. 6), a strong edge enhancement is already observed just after phase encoding has been completed ( $t=3 \mathrm{~ms}$, left part of the figure) and subsequent evolution affects the position-dependent amplitude and phase of $M_{+}$.

When the applied gradient is not parallel to the wall, distortions of the plane-wave pattern created along this gradient, with lines of constant phase constrained to be perpendicular to the walls, progressively invade the bulk of the sample. This is illustrated in Fig. 12, in which the only difference with Fig. 11 is that $G_{\mathrm{p}}$ is applied along a diagonal of the sample.

Finally, Fig. 13 displays examples of computed signals for $G_{\mathrm{p}}=0$ and a double read gradient echo for a centred read path (Fig. 13a, corresponding to $\mathrm{C}_{1} \rightarrow \mathrm{C}_{2} \rightarrow \mathrm{C}_{1}$ in Fig. 2) or an off-centred path (Fig. 13b, corresponding to $\left.\mathrm{R}_{1} \rightarrow \mathrm{R}_{2} \rightarrow \mathrm{R}_{1}\right)$. Here, $G_{\mathrm{r}}$ is aligned with the sides of the sample so that 1D maps are obtained (as in Fig. 11). The lower part of the figure (c and d) displays the corresponding 1D magnetisation maps computed at the centres of the read paths (lines), as well as the attenuated amplitudes inferred from the relevant b-values derived in section 2 for free diffusion (symbols). Except for very strong signal attenuations, the latter accurately reproduce the magneti-
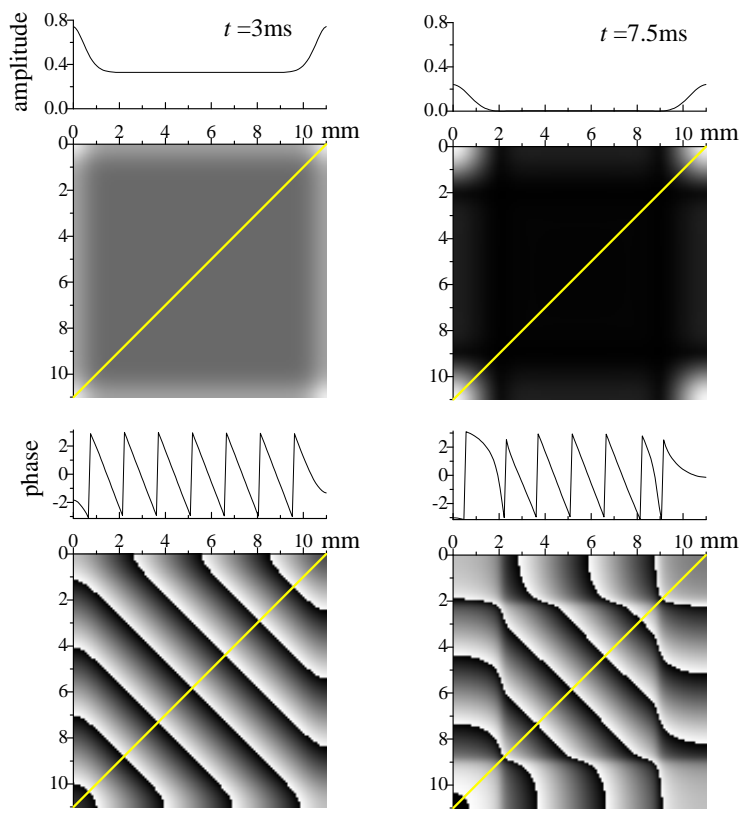

Figure 12: Computed amplitudes (top row) and phases (bottom row) of $M_{+}(t)$ are plotted as a function of position along $G_{\mathrm{p}}$, assumed here to at $45^{\circ}$ of the sides of the square sample. $2 \mathrm{D}$ maps and $1 \mathrm{D}$ profiles along the displayed diagonals are plotted. All parameters are the same as in Fig. 11.

sations obtained in the centre of the sample, away from the walls. But, in the neighbourhood of the walls, diffusion is hindered and magnetisation decay is significantly reduced. However, local phase shifts somewhat reduce the component of $M_{+}$that contributes to the signal (solid lines) with respect to its local amplitude (dashed lines). After integration over the sample, the enhanced local amplitude that is preserved near the edges contributes to a signal amplitude (shown as open symbols in Fig. 13c) that is higher than expected from a free diffusion model (Eqs. 7 and 14 , solid symbols).

The influence of edge enhancement on the reconstructed images obtained with a SLASH sequence have been quantitatively evaluated using this exact $2 \mathrm{D}$ lattice simulation in a square cell. Fig. 14 displays the reconstructed image (a) of computed data for the high-resolution $\operatorname{SLASH}\left(\mathrm{S}_{1}\right)$ sequence and the ratio $I_{2} / I_{1}$ of the two images (b). The upper parts of the plots are profiles along the indicated lines; a moderate enhancement of the amplitude in the image $(7 \%)$ is observed near the edges of the sample; the attenuation map displays variations of $I_{2} / I_{1}$ from 0.6 (at the sample centre) to 0.65 , and a sharp increase above 0.75 at the very edge of the sample. A statistical analysis of the attenuations yields $I_{2} / I_{1}=0.638 \pm 0.018$, whereas the attenuation map obtained assuming free diffusion (using, e.g., the $3 \mathrm{D}$ model) is uniform and yields $I_{2} / I_{1}=0.623$ for the same imaging parameters, which is only $2 \%$ lower. Indeed, edge enhancement and modification of the signal attenuation in a square cell may quantitatively differ 

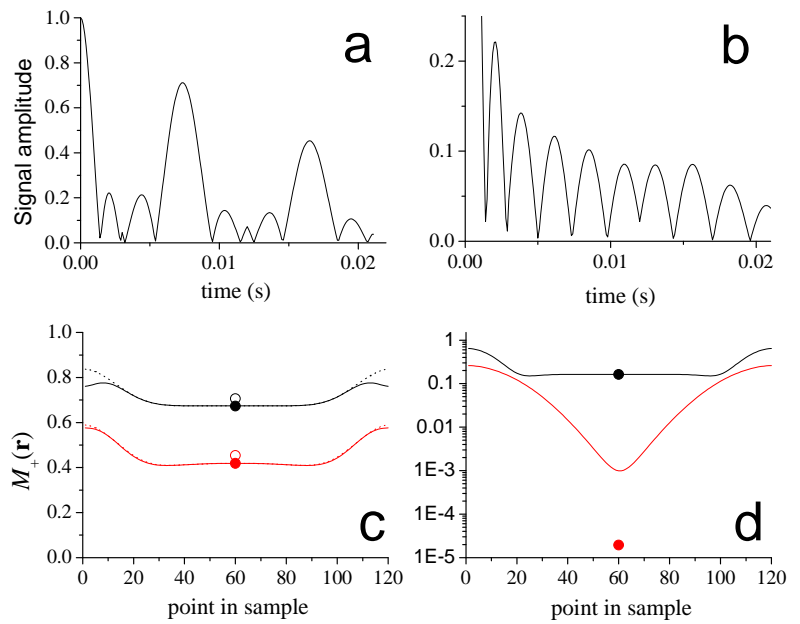

Figure 13: Computed signal amplitudes (top row) are plotted as a function of time for a double gradient echo scanning a centred interval of the k-space (Fig. a) or an off-centred one (Fig. b, see text). Bottom row: magnetisation maps at $t_{1}=7.5 \mathrm{~ms}$ (upper curves, black online) and $t_{2}=16.5 \mathrm{~ms}$ (lower curves, red online). Open symbols: signal amplitudes of Figs. a and b at the corresponding times. Closed symbols: expectations from a free diffusion model. $G_{\mathrm{r}}$ is parallel to sides of the square sample. The parameters also correspond to those of the SLASH images in Fig. 6: $T_{\text {pre }}=3 \mathrm{~ms}, T_{\mathrm{r}}=9 \mathrm{~ms} G_{\mathrm{r}}=1.3 \mathrm{mT} / \mathrm{m}\left(k_{\mathrm{r}}=12 \mathrm{~cm}\right)$, $D=1.1 \mathrm{~cm}^{2} / \mathrm{s}$.

from those obtained in a cylindrical cell. However, with a SLASH scheme, three different gradient orientations are probed: parallel, perpendicular, and at $45^{\circ}$ with respect to the walls of the square cell, so that different effects of distortions of phase maps in the bulk of the gas due to boundary conditions (see Fig. 12) are actually taken into account.

To summarise the results of these simple 2D simulations, weak and localised image distortions (edge enhance-

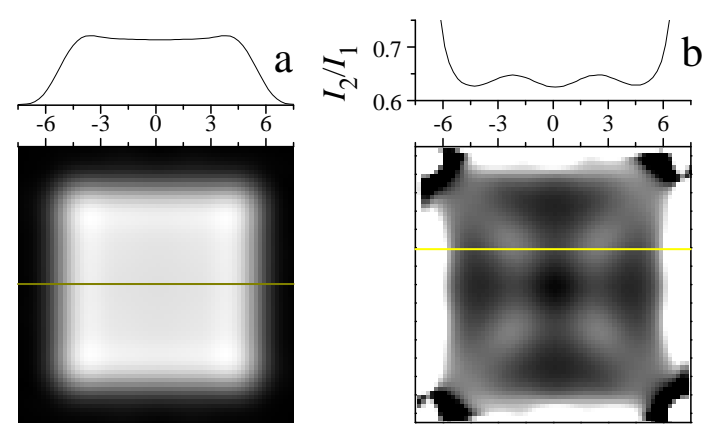

Figure 14: Images and profiles obtained using the exact $2 \mathrm{D}$ simulation with the sequence $\mathrm{S}_{1}$ in a $11 \mathrm{~mm}$ square box for $D=1.1 \mathrm{~cm}^{2} / \mathrm{s}$. Figure a: image $I_{1}$ reconstructed from the first echo. Figure b: ratio $I_{2} / I_{1}$ of the images from the two echoes. ment) and a mild decrease of the average inter-image attenuation have been found for our SLASH imaging conditions and samples. These simulations provide a flexible and fast tool to quantify these effects and to validate the use of the simplified 3D method of Section 3.2 to compute the effect of concomitant gradients in our experiments.

\section{Apodisation and point spread functions in double-cross SLASH and FLASH schemes}

Three apodisation functions have been used in image reconstructions: the usual Hanning window $w_{\mathrm{H}}$ and broader sine-based apodisation windows $w_{1}$ and $w_{2}$ suggested in [53]:

$$
\begin{aligned}
w_{\mathrm{H}}\left(p, N_{\mathrm{p}}\right) & =\sin ^{2}\left[\pi p /\left(N_{\mathrm{p}}+1\right)\right] \\
w_{1}\left(p, N_{\mathrm{p}}\right) & =\sin \left[\pi p /\left(N_{\mathrm{p}}+1\right)\right] \\
w_{2}\left(p, N_{\mathrm{p}}\right) & =2 \sin \left[\pi p /\left(N_{\mathrm{p}}+1\right)\right]-\sin ^{2}\left[\pi p /\left(N_{\mathrm{p}}+1\right)\right] .
\end{aligned}
$$

The efficiency of these apodisation functions to reduce Gibbs truncation artefacts is conveniently discussed using Fig. 15, that displays the associated point spread functions $(\mathrm{PSF}$ ) for a $1 \mathrm{D} \times 1 \mathrm{D}$ factorised apodisation ( $\mathrm{a}$ and $\mathrm{b}$, FLASH reconstruction scheme) and for a $2 \mathrm{D}$ radial apodisation (c and d, SLASH reconstruction scheme). Without apodisation, the PSFs display nodes and secondary lobes that directly reflect the square or near-circular symmetry of the acquired k-space (Fig. 15a and c). Amplitude profiles are displayed in Figs. $15 \mathrm{~b}$ and $\mathrm{d}$ for selected situations. Without apodisation, the narrowest and most oscillating profiles are obtained, corresponding to the plotted images. For the FLASH reconstruction scheme, the profiles along the encoding direction (the solid line, corresponding to a sinc function) and along a diagonal (the dashed line) differ in width and shape. As is well known, the oscillations are efficiently reduced when a Hanning apodisation $w_{\mathrm{H}}$ is used, but a significant broadening $(\times 1.6)$ of the PSF inevitably results. All widths and amplitudes are listed in Table 4 . For the SLASH reconstruction scheme, the isotropic PSF profile is the (amplitude) Airy function $2 J_{1}\left(k_{\mathrm{p}} x\right) / k_{\mathrm{p}} x$ when no apodisation is performed (image and narrowest curve in the figure). Fig. 15d also displays the PSF profiles obtained when applying $w_{2}, w_{1}$, and $w_{\mathrm{H}}$. They exhibit increasing widths and decreasing oscillations. The amplitude of the first minimum is evenly distributed in a uniform ring and it is more shallow than the localised minima of the square pattern. Furthermore, oscillations decay more rapidly with increasing distance. Gibbs truncation artefacts are thus usually less prominent in SLASH images, and therefore a satisfactory trade-off between the broadening of the PSF and its oscillations can often be obtained using $w_{1}$ or $w_{2}$ instead of $w_{\mathrm{H}}$, depending on the contrasts in the imaged object. 

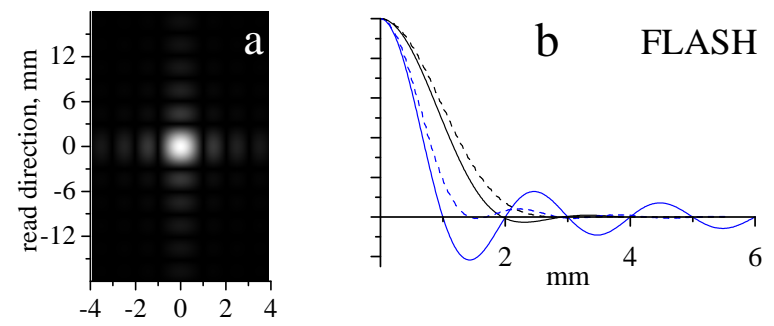

Encode direction, $\mathrm{mm}$
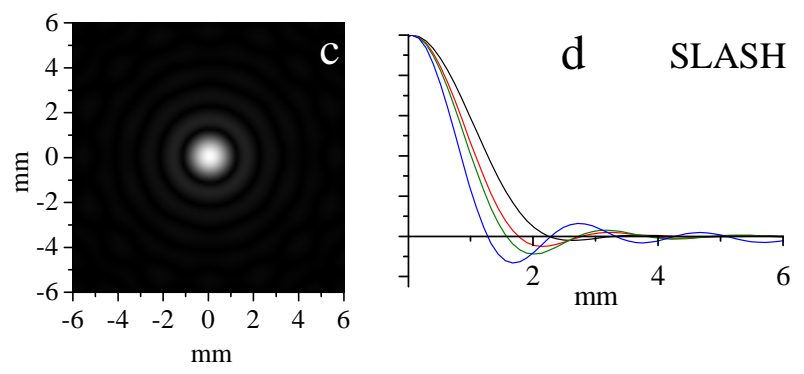

Figure 15: Computed PSFs corresponding to truncation in k-space. Figures a and b: FLASH reconstruction scheme for maximum $k$ values $(k= \pm \pi / \delta)$ corresponding to resolutions $\delta_{\mathrm{r}}=3 \mathrm{~mm}$ and $\delta_{\mathrm{p}}=1 \mathrm{~mm}$. Figures $\mathrm{c}$ and $\mathrm{d}$ : SLASH reconstruction for the same parameters, that are those of the high-resolution acquisitions of Fig. 6. Images a and c: 2D PSFs resulting from sharp truncation, that yields sinc function variations along the axes (for image a, with profiles in Fig. b) or an isotropic Airy function variation (for image c, with profiles in Fig. d). Figure b: PSF profiles (FLASH scheme) along the phase encode axis (solid lines) or a diagonal (dotted lines), without apodisation (thin blue curves) or with Hanning apodisation (thick black curves). Figure d: PSF profiles (SLASH scheme) for various apodisations. All widths and oscillation characteristics are listed in Table 4.

\section{References}

[1] A. Haase, J. Frahm, D. Matthaei, W. Hanicke, et al. 'FLASH imaging. Rapid NMR imaging using low flipangle pulses.' J. Magn. Reson. (1969), 67(2):258266 (1986). doi:DOI: 10.1016/0022-2364(86)90433-6.

[2] L. Zhao, R. Mulkern, C.-H. Tseng, D. Williamson, et al. 'Gradient-Echo Imaging Considerations for Hyperpolarized 129Xe MR.' J. Magn. Reson. $B, \quad 113(2): 179 \quad-183$ (1996). doi:DOI: 10.1006/jmrb.1996.0173.

[3] B. Saam, D. A. Yablonskiy, D. S. Gierada, and M. S. Conradi. 'Rapid imaging of hyperpolarized gas using EPI.' Magn. Reson. Med., 42(3):507-514 (1999). doi:10.1002/(SICI)15222594(199909)42:3¡507::AID-MRM13¿3.0.CO;2-U.

[4] D. S. Gierada, B. Saam, D. Yablonskiy, J. D. Cooper, et al. 'Dynamic echo planar MR imaging of lung ventilation with hyperpolarized $3 \mathrm{He}$ in normal subjects

\begin{tabular}{|l|c|c|c|c|}
\hline \multicolumn{1}{|c|}{ sequence } & window & FWHM & $1^{\text {st }} \min$ & $1^{\text {st }} \max$ \\
\hline FLASH & none & 1.218 & -.217 & .128 \\
FLASH & $w_{H}$ & 1.954 & -.027 & .009 \\
FLASH (diag) & none & 1.360 & -.009 & .041 \\
FLASH (diag) & $w_{H}$ & 2.164 & - & - \\
\hline SLASH & none & 1.40 & -.133 & .064 \\
SLASH & $w_{2}$ & 1.682 & -.088 & .031 \\
SLASH & $w_{1}$ & 1.805 & -.051 & .019 \\
SLASH & $w_{H}$ & 2.132 & -.021 & .006 \\
\hline
\end{tabular}

Table 4: Table of full widths at half-maximum (in $\mathrm{mm}$ ) and of the relative amplitudes of the first minimum and following maximum for the profiles plotted in the righthand side part of Fig 15. For the FLASH reconstruction scheme, FWHMs are multiplied by 1.6 when the Hanning window $w_{\mathrm{H}}$ is applied. For SLASH reconstruction, FWHMs are multiplied by $1.2,1.29$, and 1.52 (compared to that of the Airy function) for increasing degrees of apodisation.

and patients with severe emphysema.' NMR Biomed., 13(4):176-181 (2000).

[5] E. Durand, G. Guillot, L. Darrasse, G. Tastevin, et al. 'CPMG measurements and ultrafast imaging in human lungs with hyperpolarized helium-3 at low field (0.1 T).' Magn. Reson. Med., 47(1):75-81 (2002). doi:10.1002/mrm.10047.

[6] J. R. Owers-Bradley, M. S. Fichele, A. Bennattayalah, C. J. Smith McGloin, et al. 'MR tagging of human lungs using hyperpolarized 3He gas.' J. Magn. Reson. Imaging, 17(1):142-146 (2003). doi: 10.1002/jmri.10226.

[7] C. Bidinosti, J. Choukeife, G. Tastevin, P.-J. Nacher, et al. 'MRI of the lung using hyperpolarized $3 \mathrm{He}$ at very low magnetic field (3 mT).' Magn. Reson. Mater. Phy., 16:255-258 (2004).

[8] J. M. Wild, K. Teh, N. Woodhouse, M. N. Paley, et al. 'Steady-state free precession with hyperpolarized 3He: Experiments and theory.' J. Magn. Reson., 183(1):13 - 24 (2006). doi:10.1016/j.jmr.2006.07.015.

[9] M. H. Deppe, K. Teh, J. Parra-Robles, K. J. Lee, et al. 'Slice profile effects in $2 \mathrm{D}$ slice-selective MRI of hyperpolarized nuclei.' J. Magn. Reson., 202(2):180 - 189 (2010). doi:10.1016/j.jmr.2009.11.003.

[10] M. Salerno, J. R. Brookeman, E. E. de Lange, and J. P. Mugler. 'Hyperpolarized $3 \mathrm{He}$ lung imaging at 0.5 and 1.5 Tesla: A study of susceptibility-induced effects.' Magn. Reson. Med., 53(1):212-216 (2005). doi:10.1002/mrm.20329.

[11] J. Parra-Robles, W. D. Viqueira, X. Xu, A. Ouriadov, et al. 'Theoretical prediction and experimental measurement of the field dependence of the apparent 
transverse relaxation of hyperpolarized noble gases in lungs.' J. Magn. Reson., 192(1):85-91 (2008). doi: 10.1016/j.jmr.2008.02.009.

[12] M. H. Deppe, J. Parra-Robles, S. Ajraoui, S. R. Parnell, et al. 'Susceptibility effects in hyperpolarized 3He lung MRI at $1.5 \mathrm{~T}$ and 3T.' J. Magn. Reson. Imaging, 30(2):418-423 (2009). doi: 10.1002/jmri.21852.

[13] C. Wang, T. A. Altes, J. P. Mugler, G. W. Miller, et al. 'Assessment of the lung microstructure in patients with asthma using hyperpolarized $3 \mathrm{He}$ diffusion MRI at two time scales: Comparison with healthy subjects and patients with COPD.' J. Magn. Reson. Imaging, 28(1):80-88 (2008). doi: 10.1002/jmri.21408.

[14] S. Fain, M. L. Schiebler, D. G. McCormack, and G. Parraga. 'Imaging of lung function using hyperpolarized helium-3 magnetic resonance imaging: Review of current and emerging translational methods and applications.' J. Magn. Reson. Imaging, 32(6):1398-1408 (2010). doi:10.1002/jmri.22375.

[15] X. Xu, M. Boudreau, A. Ouriadov, and G. E. Santyr. 'Mapping of $3 \mathrm{He}$ apparent diffusion coefficient anisotropy at sub-millisecond diffusion times in an elastase-instilled rat model of emphysema.' Magn. Reson. Med., 67(4):1146-1153 (2012). doi: 10.1002/mrm.23098.

[16] M. Boudreau, X. Xu, and G. E. Santyr. 'Measurement of 129xe gas apparent diffusion coefficient anisotropy in an elastase-instilled rat model of emphysema.' Magn. Reson. Med. (2012). doi: $10.1002 / \mathrm{mrm} .24224$.

[17] J. Schmiedeskamp, W. Heil, E. W. Otten, R. K. Kremer, et al. 'Paramagnetic relaxation of spin polarized He3 at bare glass surfaces.' Eur. Phys. J. D, 38:427438 (2006).

[18] A. Deninger, W. Heil, E. W. Otten, M. Wolf, et al. 'Paramagnetic relaxation of spin polarized He3 at coated glass walls.' Eur. Phys. J. D, 38:439-443 (2006).

[19] C. Gemmel, W. Heil, S. Karpuk, K. Lenz, et al. 'Ultra-sensitive magnetometry based on free precession of nuclear spins.' Eur. Phys. J. D, 57(3):303-320 (2010). doi:10.1140/epjd/e2010-00044-5.

[20] C. P. Bidinosti, J. Choukeife, P.-J. Nacher, and G. Tastevin. 'In vivo NMR of hyperpolarized $3 \mathrm{He}$ in the human lung at very low magnetic fields.' $J$. Magn. Reson., 162(1):122 - 132 (2003). doi:DOI: 10.1016/S1090-7807(02)00198-2.
[21] G. Tastevin and P. J. Nacher. 'NMR measurements of hyperpolarized He-3 gas diffusion in high porosity silica aerogels.' J. Chem. Phys., 123(6):064506 (2005). doi:10.1063/1.1997130.

[22] R. H. Acosta, P. Blumler, K. Mannemann, and H.W. Spiess. 'Mixture and dissolution of laser polarized noble gases: Spectroscopic and imaging applications.' Prog. Nucl. Magn. Reson. Spectrosc., 66(0):40 - 69 (2012). doi:10.1016/j.pnmrs.2012.03.003.

[23] J. M. Wild, M. N. Paley, M. Viallon, W. G. Schreiber, et al. ' $\mathrm{k}$-Space filtering in 2D gradient-echo breathhold hyperpolarized 3He MRI: Spatial resolution and signal-to-noise ratio considerations.' Magn. Reson. Med., 47(4):687-695 (2002). doi:10.1002/mrm.10134.

[24] J. Parra-Robles, A. R. Cross, and G. E. Santyr. 'Theoretical signal-to-noise ratio and spatial resolution dependence on the magnetic field strength for hyperpolarized noble gas magnetic resonance imaging of human lungs.' Med. Phys., 32(1):221-229 (2005). doi:10.1118/1.1833593.

[25] J. P. Dugas, J. R. Garbow, D. K. Kobayashi, and M. S. Conradi. 'Hyperpolarized 3He MRI of mouse lung.' Magn. Reson. Med., 52(6):1310-1317 (2004). doi:10.1002/mrm.20300.

[26] P. T. Callaghan. Principles of Nuclear Magnetic Resonance Microscopy. Oxford University Press, New York (1991).

[27] J. Pipe. 'Motion correction with PROPELLER MRI: Application to head motion and freebreathing cardiac imaging.' Magn. Reson. Med., 42(5):963-969 (1999). doi:10.1002/(SICI)15222594(199911)42:5¡963::AID-MRM17¡3.0.CO;2-L.

[28] K. Arfanakis, A. A. Tamhane, J. G. Pipe, and M. A. Anastasio. 'K-space undersampling in PROPELLER imaging.' Magn. Reson. Med., 53(3):675-683 (2005). doi:10.1002/mrm.20380.

[29] S. Kojima, S. Morita, E. Ueno, M. Hirata, et al. 'Aliasing artifacts with the BLADE technique: Causes and effective suppression.' J. Magn. Reson. Imaging, 33(2):432-440 (2011). doi: 10.1002/jmri.22465.

[30] B. Saam, N. Drukker, and W. Happer. 'Edge enhancement observed with hyperpolarized 3He.' Chem. Phys. Lett., 263(3-4):481 - 487 (1996). doi: DOI: 10.1016/S0009-2614(96)01238-9.

[31] Y. Q. Song, B. M. Goodson, B. Sheridan, T. M. de Swiet, et al. 'Effects of diffusion on magnetic resonance imaging of laser-polarized xenon gas.' J. Chem. Phys., 108(15):6233-6239 (1998). doi: $10.1063 / 1.476030$. 
[32] T. M. de Swiet and P. N. Sen. 'Decay of nuclear magnetization by bounded diffusion in a constant field gradient.' J. Chem. Phys., 100(8):5597-5604 (1994). doi:10.1063/1.467127.

[33] T. M. de Swiet. 'Diffusive edge enhancement in imaging.' J. Magn. Reson. B, 109(1):12 - 18 (1995). doi: DOI: $10.1006 /$ jmrb.1995.1141.

[34] J. Stepisnik, A. Duh, A. Mohoric, and I. Sersa. 'MRI Edge Enhancement as a Diffusive Discord of Spin Phase Structure.' J. Magn. Reson., 137(1):154 - 160 (1999). doi:DOI: 10.1006/jmre.1998.1678.

[35] A. Sukstanskii, J. Ackerman, and D. Yablonskiy. 'Effects of barrier-induced nuclear spin magnetization inhomogeneities on diffusion-attenuated MR signal.' Magn. Reson. Med., 50(4):735-742 (2003). doi: 10.1002/mrm.10586.

[36] D. G. Norris and J. M. Hutchison. 'Concomitant magnetic field gradients and their effects on imaging at low magnetic field strengths.' Magn. Reson. Imaging, 8(1):33-37 (1990).

[37] D. A. Yablonskiy, A. L. Sukstanskii, and J. J. Ackerman. 'Image artifacts in very low magnetic field MRI: The role of concomitant gradients.' J. Magn. Reson., 174(2):279 - 286 (2005). doi: 10.1016/j.jmr.2005.02.016.

[38] P. J. Nacher, M. Pelissier, and G. Tastevin. 'Design and test of a magnet and gradient system for hyperpolarised gas lung MRI at ultra-low field.' In 'Proc. Intl. Soc. Mag. Reson. Med. 15 (2007),' page 3290 (2007).

[39] E. Baudin, K. Safiullin, S. W. Morgan, and P. J. Nacher. 'An active feedback scheme for low field NMR experiments.' J. Phys.: Conf. Ser., 294(1):012009 (2011).

[40] J. Choukeife, X. Maitre, P. Nacher, and G. Tastevin. 'On-site production of hyperpolarised helium-3 gas for lung MRI.' In 'Proc. Intl. Soc. Mag. Reson. Med. 11 (2003),' page 1391 (2003).

[41] H. Marshall, S. Ajraoui, M. H. Deppe, J. ParraRobles, et al. 'K-space filter deconvolution and flip angle self-calibration in $2 \mathrm{D}$ radial hyperpolarised $3 \mathrm{He}$ lung MRI.' NMR Biomed., 25(2):389-399 (2012). doi: 10.1002/nbm.1766.

[42] J. D. O'Sullivan. 'A fast sinc function gridding algorithm for Fourier inversion in computer tomography.' IEEE transactions on medical imaging, 4(4):200-207 (1985). doi:10.1109/TMI.1985.4307723.

[43] S. S. Kaushik, Z. I. Cleveland, G. P. Cofer, G. Metz, et al. 'Diffusion-weighted hyperpolarized 129Xe MRI in healthy volunteers and subjects with chronic obstructive pulmonary disease.' Magn. Reson. Med., 65(4):1154-1165 (2011). doi:10.1002/mrm.22697.

[44] S. Fichele, M. Paley, N. Woodhouse, P. Griffiths, et al. 'Investigating He-3 diffusion NMR in the lungs using finite difference simulations and in vivo PGSE experiments.' J. Magn. Reson., 167(1):1-11 (2004). doi:10.1016/j.jmr.2003.10.019.

[45] S. Fichele, M. Paley, N. Woodhouse, P. Griffiths, et al. 'Finite-difference simulations of He-3 diffusion in 3D alveolar ducts: Comparison with the "cylinder model".' Magn. Reson. Med., 52(4):917-920 (2004). doi:10.1002/mrm.20213.

[46] J. Parra-Robles, S. Ajraoui, M. H. Deppe, S. R. Parnell, et al. 'Experimental investigation and numerical simulation of He-3 gas diffusion in simple geometries: Implications for analytical models of He-3 MR lung morphometry.' J. Magn. Reson., 204(2):228-238 (2010). doi:10.1016/j.jmr.2010.02.023.

[47] T. Enss, S. Ahn, and W. S. Warren. 'Visualizing the dipolar field in solution NMR and MR imaging: three dimensional structure simulations.' Chem. Phys. Lett., 305:101-108 (1999).

[48] J. Jeener. 'Dynamical Effects of the Dipolar Field Inhomogeneities in High-Resolution NMR: Spectral Clustering and Instabilities.' Phys. Rev. Lett., 82(8):1772-1775 (1999).

[49] J. Jeener. 'Collective Effects in Liquid NMR: Dipolar Field and Radiation Damping.' In D. M. Grant and R. K. Harris, editors, 'Encyclopedia of NMR,' volume 9, pages 642-679. Wiley (2002).

[50] P. J. Nacher, N. Piegay, F. Marion, and G. Tastevin. 'NMR instabilities in highly magnetised liquid helium solutions.' J. Low Temp. Phys., 126(1-2):145150 (2002). doi:10.1023/A:1013787405665.

[51] M. E. Hayden, E. Baudin, G. Tastevin, and P. J. Nacher. 'NMR Time Reversal as a Probe of Incipient Turbulent Spin Dynamics.' Phys. Rev. Lett., 99(13):137602 (2007). doi: 10.1103/PhysRevLett.99.137602.

[52] E. Baudin. Non linear NMR spin dynamics and time-reversal experiments in hyperpolarized $3 \mathrm{He}-4 \mathrm{He}$ liquid mixtures at low temperature. Ph.D. thesis, Université Pierre et Marie Curie, Paris (2010). http://tel.archives-ouvertes.fr/tel-00623612.

[53] Mahesh, R. Gupta, R. Kalyanaraman, S. Rao, et al. 'Truncation artifact reduction in MRI with a new class of filters.' In 'Proc. 7th Intl. Soc. Mag. Reson. Med.', page 178 (1999). 